Pacific

Journal of

Mathematics

CATEGORIFICATION OF A PARABOLIC HECKE MODULE VIA SHEAVES ON MOMENT GRAPHS

MARTINA LANINI

Volume $271 \quad$ No. 2

October 2014 


\title{
CATEGORIFICATION OF A PARABOLIC HECKE MODULE VIA SHEAVES ON MOMENT GRAPHS
}

\author{
MARTINA LANINI
}

\begin{abstract}
We investigate certain categories, associated by Fiebig with the geometric representation of a Coxeter system, via sheaves on Bruhat graphs. We modify Fiebig's definition of translation functors in order to extend it to the singular setting and use it to categorify a parabolic Hecke module. As an application we obtain a combinatorial description of indecomposable projective objects of (truncated) noncritical singular blocks of (a deformed version of) category 0 , using indecomposable special modules over the structure algebra of the corresponding Bruhat graph.
\end{abstract}

\section{Introduction}

A typical problem in the representation theory of Kac-Moody algebras is to understand the composition series of standard objects in the corresponding category $\mathbb{O}$ of Bernstein, I. Gelfand and S. Gelfand [Bernstein et al. 1976]. In the case of a standard object lying in a regular block, this question is the core of the Kazhdan-Lusztig theory, and the answer is known to be given by the Kazhdan-Lusztig polynomials evaluated at the identity. If we consider a singular block, we only have to replace these polynomials by their parabolic analogue. In the case of a principal block, this fact was conjectured in [Kazhdan and Lusztig 1979] and proved in several steps in [Kazhdan and Lusztig 1980; Beilinson and Bernstein 1981; Brylinski and Kashiwara 1980]. A fundamental role in the proof of the Kazhdan-Lusztig conjecture was played by the geometric interpretation of the problem in terms of perverse sheaves and intersection cohomology complexes. In particular, one could study certain properties of the Hecke algebra in the category of equivariant perverse sheaves on the corresponding flag variety.

An alternative way to attack the Kazhdan-Lusztig conjecture is via Soergel bimodules, which provide a combinatorial realisation of projective objects in category 0 . The combinatorial description of indecomposable projective objects we present in this paper is an analogue of the combinatorial construction of [Soergel 1990]

MSC2010: 17B67, $20 \mathrm{C} 08$.

Keywords: sheaves on moment graphs, parabolic Hecke module. 
(introduced at first for finite-dimensional Lie algebras). The Soergel bimodule approach to the Kazhdan-Lusztig conjecture recently led to an algebraic proof of it [Elias and Williamson 2014].

The procedure of considering a complicated object, such as a category, in order to understand a simpler one is motivated by the fact that the extra structure can provide us with new tools and allow us to prove and hopefully generalise certain phenomena that are difficult to address directly.

Deodhar [1987] associated with any Coxeter system $(\mathcal{W}, \mathscr{S})$ and any subset of the set of simple reflections $J \subseteq \mathscr{Y}$ the parabolic Hecke module $\boldsymbol{M}^{J}$. The aim of this paper is to give a categorification of this module for any $J$ generating a finite subgroup.

We have followed the definition of categorification of $\boldsymbol{M}^{J}$ in [Mazorchuk and Stroppel 2005, Remark 7.8], which is actually a weak categorification. This could be strengthened to a proper categorification by presenting the result as a 2representation of some 2-category (see [Mazorchuk 2012, Sections 1-3] for various levels of categorification and Remark 5.9 of this paper for a more precise statement). In [Mazorchuk and Stroppel 2008], the authors properly categorify induced cell modules (in the finite case), which is a huge step outside the parabolic Hecke module (the latter being just a special case).

If $\mathcal{W}$ is a Weyl group, there is a partial flag variety $Y$ corresponding to the set $J$, equipped with an action of a maximal torus $T$, and as for the regular case, one possible categorification is given by the category of $B$-equivariant perverse sheaves on $Y$. Our goal is to describe a general categorification, which can be defined also in the case in which there is no geometry available. In order to do this, our main tools will be Bruhat moment graphs and sheaves on them. We will see how these objects come naturally into the picture.

Moment graphs appeared for the first time in [Goresky et al. 1998] as 1-skeletons of actions of tori on complex algebraic varieties. In particular, Goresky, Kottwitz and MacPherson were able to describe explicitly the equivariant cohomology of these varieties using only the data encoded in the underlying moment graphs. Inspired by this result, Braden and MacPherson [2001] could study the equivariant intersection cohomology of a complex algebraic variety equipped with a Whitney stratification, stable with respect to the torus action. In order to do so, they introduced the notion of sheaves on moment graphs and, in particular, of canonical sheaves. We will refer to this class of sheaves as Braden-MacPherson sheaves, or BMP sheaves.

Even if moment graphs arose originally from geometry, Fiebig [2008b] observed that it is possible to give an axiomatic definition of them. In particular, he associated a moment graph to any Coxeter datum $(\mathcal{W}, \mathcal{Y}, J)$ as above and in the case of $J=\varnothing$, he used it to give an alternative construction of Soergel's category of bimodules associated to a reflection-faithful representation of (W, $\mathscr{Y})$ (see [Fiebig 2008b]). 
(We refer the reader to [Williamson 2011] for the singular version of Soergel's bimodules.) The indecomposable objects of the category defined by Fiebig are precisely the BMP sheaves that, if $\mathscr{W}$ is a Weyl group, are related to the intersection cohomology complexes, the simple objects in the category of perverse sheaves. A fundamental step in Fiebig's realisation of this category were translation functors, whose definition we extend to the parabolic setting (see p. 426).

The paper is organised as follows:

In Section 2 we recall the definition of the parabolic Hecke module $\boldsymbol{M}^{J}$ and the fact that it is the unique free $\mathbb{Z}\left[v, v^{-1}\right]$-module having rank $|\mathcal{W} /\langle J\rangle|$ equipped with a certain structure of a module over the Hecke algebra $\boldsymbol{H}$. This structure is described in terms of the action of the Kazhdan-Lusztig basis elements $\underline{H}_{s}$, for $s \in \mathscr{Y}$. Then by a categorification of $\boldsymbol{M}^{J}$ (as in [Mazorchuk and Stroppel 2005, Remark 7.8]) we mean a category $\mathscr{C}$, which is exact in the sense of Quillen [1973], together with an autoequivalence $G$ and exact functors $\left\{F_{s}\right\}_{s \in \mathscr{S}}$ that provide the Grothendieck group [ $\mathscr{C}]$ with the structure of a $\mathbb{Z}\left[v, v^{-1}\right]$-module and $\boldsymbol{H}$-module, such that there exists an isomorphism from $[\mathscr{C}]$ to the parabolic module, satisfying certain compatibility conditions with these functors coming from the defining properties of $\boldsymbol{M}^{J}$ (see definition on p. 420).

In the third section we introduce the objects we will be dealing with in the rest of the paper. In particular, we review basic concepts of the theory of moment graphs and sheaves on them.

Section 4 is about $\mathbb{Z}$-graded modules over $\mathscr{E}^{J}$, the structure algebra of a parabolic Bruhat graph. In particular for any $s \in \mathscr{Y}$, we define the translation functor ${ }^{s} \theta$ and define the category $\mathscr{H}^{J}$ of special $\mathscr{L}^{J}$-modules. By definition, this category is stable under the shift in degree that we denote by $\langle\cdot\rangle$ and under ${ }^{s} \theta$ for all $s \in \mathscr{T}$.

In Section 5 we study certain subquotients of objects in $\mathscr{H}^{J}$, and this allows us to define an exact structure on $\mathscr{H}^{J}$ and hence to state our main theorem:

Theorem 5.8. The category $\mathscr{H}^{J}$ special $\mathscr{L}^{J}$-modules together with the shift in degree $\langle-1\rangle$ and (shifted) translation functors is a categorification of the parabolic Hecke module $\boldsymbol{M}^{J}$.

Section 6 is devoted to the proof of this theorem. First we show that ${ }^{s} \theta \circ\langle 1\rangle$ is an exact functor (Lemma 6.1). Secondly we define the character map $h^{J}:\left[\mathscr{H}^{J}\right] \rightarrow \boldsymbol{M}^{J}$ and prove that the functors $\langle-1\rangle$ and ${ }^{s} \theta \circ\langle 1\rangle, s \in \mathscr{Y}$, satisfy the desired compatibility condition (Proposition 6.2). We conclude then by showing that the character map is an isomorphism of $\mathbb{Z}\left[v, v^{-1}\right]$-modules (Lemma 6.3 and Lemma 6.6).

Section 7 is about the categorification of a certain injective map of $\boldsymbol{H}$-modules $i: \boldsymbol{M}^{J} \hookrightarrow \boldsymbol{H}$, which allows us to see the category $\mathscr{H}^{J}$ as a subcategory of $\mathscr{H}^{\varnothing}$. More precisely, we define an exact functor $I: \mathscr{H}^{J} \rightarrow \mathscr{H}^{\varnothing}$ such that the following diagram commutes: 


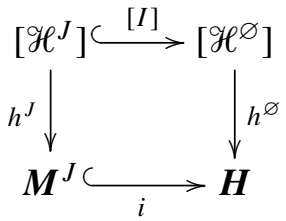

In order to construct and investigate the functor $I$, we give a realisation of $\mathscr{H}^{J}$ via BMP sheaves (Proposition 6.5) and then use Fiebig's idea [2008b] of interchanging global and local viewpoints.

In the last section we discuss briefly the relationship between $\mathscr{H}^{J}$ and noncritical blocks of an equivariant version of category 0 for symmetrisable Kac-Moody algebras. In particular, we show that the indecomposable projective objects of a truncated, noncritical block $\mathrm{O}_{R, \Lambda} \leq \nu$ are combinatorially described by indecomposable modules in $\mathscr{H}^{J}$, with $J$ depending on $\Lambda$ (Proposition 8.3).

\section{Hecke modules}

Here we recall some classical constructions, following [Soergel 1997]. We close the section by defining the concept of categorification of the parabolic Hecke module $\boldsymbol{M}^{J}$.

Hecke algebra. The Hecke algebra associated to a Coxeter system $(\mathscr{W}, \mathscr{Y})$ is nothing but a quantisation of the group ring $\mathbb{Z}[W]$. Let $\leq$ be the Bruhat order on $\mathscr{W}$ and $\ell: \mathscr{W} \rightarrow \mathbb{Z}$ be the length function associated to $\mathscr{Y}$. Denote by $\mathscr{L}:=\mathbb{Z}\left[v, v^{-1}\right]$ the ring of Laurent polynomials in the variable $v$ over $\mathbb{Z}$.

Definition 2.1. The Hecke algebra $\boldsymbol{H}=\boldsymbol{H}(\mathcal{W}, \mathscr{Y})$ is the free $\mathscr{L}$-module having basis $\left\{H_{x} \mid x \in \mathscr{W}\right\}$, subject to the following relations, for $x \in \mathscr{W}, s \in \mathscr{Y}$ :

$$
H_{s} H_{x}= \begin{cases}H_{s x} & \text { if } s x>s, \\ \left(v^{-1}-v\right) H_{x}+H_{s x} & \text { if } s x<x,\end{cases}
$$

It is well known that this defines an associative $\mathscr{L}$-algebra [Humphreys 1990].

It is easy to verify that $H_{x}$ is invertible for any $x \in \mathcal{W}$, and this allows us to define an involution on $\boldsymbol{H}$, that is, the unique ring homomorphism ${ }^{-}: \boldsymbol{H} \rightarrow \boldsymbol{H}$ such that $\bar{v}=v^{-1}$ and $\bar{H}_{x}=\left(H_{x^{-1}}\right)^{-1}$.

Kazhdan and Lusztig [1979] showed the existence of another basis $\left\{\underline{H}_{x}\right\}$ for $\boldsymbol{H}$, the so-called Kazhdan-Lusztig basis, that they used to define complex representations of the Hecke algebra and hence of the Coxeter group. The entries of the change of basis matrix are given by a family of polynomials in $\mathbb{Z}[v]$, which are called Kazhdan-Lusztig polynomials. There are many different normalisations of this basis appearing in the literature. The one we adopt, following [Soergel 1997], is determined by Theorem 2.2 (see Remark 2.3). 
Parabolic Hecke modules. Deodhar [1987] generalised this construction to the parabolic setting in the following way. Let $\mathscr{W}, \mathscr{S}$ and $\boldsymbol{H}$ be as above. Fix a subset $J \subseteq \mathscr{Y}$ and denote by $\mathscr{W}_{J}=\langle J\rangle$ the subgroup of $\mathscr{W}$ generated by $J$. Since $\left(W_{J}, J\right)$ is also a Coxeter system, it makes sense to consider its Hecke algebra $\boldsymbol{H}_{J}=\boldsymbol{H}\left(\mathcal{W}_{J}, J\right)$.

For any simple reflection $s \in \mathscr{Y}$, the element $H_{s}$ satisfies the quadratic relation $\left(H_{s}\right)^{2}=\left(v^{-1}-v\right) H_{s}+H_{e}$, that is, $\left(H_{s}+v\right)\left(H_{s}-v^{-1}\right)=0$. If $u \in\left\{v^{-1},-v\right\}$, we may define a map of $\mathscr{L}$-modules $\varphi_{u}: \boldsymbol{H}_{J} \rightarrow \mathscr{L}$ by $H_{s} \mapsto u$. In this way, $\mathscr{L}$ is endowed with the structure of a $\boldsymbol{H}_{J}$-bimodule, which we denote by $\mathscr{L}(u)$.

The parabolic Hecke modules are then defined as $\boldsymbol{M}^{J}:=\boldsymbol{H} \otimes_{\boldsymbol{H}_{J}} \mathscr{L}\left(v^{-1}\right)$ and $\boldsymbol{N}^{J}:=\boldsymbol{H} \otimes_{\boldsymbol{H}_{J}} \mathscr{L}(-v)$. As in the Hecke algebra case, it is possible to define an involutive automorphism of these modules. Namely,

$$
\begin{aligned}
\overline{:} \boldsymbol{H} \otimes_{\boldsymbol{H}_{J}} \mathscr{L}(u) & \rightarrow \boldsymbol{H} \otimes_{\boldsymbol{H}_{J}} \mathscr{L}(u), \\
H \otimes a & \mapsto \bar{H} \otimes \bar{a} .
\end{aligned}
$$

For $u \in\left\{v^{-1},-v\right\}$, let $H_{w}^{J, u}:=H_{w} \otimes 1 \in \mathscr{L}(u) \otimes_{\boldsymbol{H}_{J}} \boldsymbol{H}$. Denote by $\mathscr{W}^{J}$ the set of minimal length representatives of $\mathscr{W} / \mathscr{W}_{J}$.

Theorem 2.2 [Deodhar 1987].

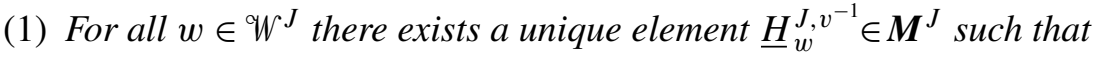

(a) $\underline{\bar{H}}_{w}^{J, v^{-1}}=\underline{H}_{w}^{J, v^{-1}}$, and

(b) $\underline{H}_{w}^{J, v^{-1}}=\sum_{y \in \mathscr{W}^{J}} m_{y, w}^{J} H_{y}^{J, v^{-1}}$,

where the $m_{y, w}^{J}$ are such that $m_{w, w}^{J}=1$ and $m_{y, w}^{J} \in v \mathbb{Z}[v]$ if $y \neq w$.

(2) For all $w \in \mathscr{W}^{J}$ there exists a unique element $\underline{H}_{w}^{J,-v} \in N^{J}$ such that

(a) $\underline{\bar{H}}_{w}^{J,-v}=\underline{H}_{w}^{J,-v}$, and

(b) $\underline{H}_{w}^{J,-v}=\sum_{y \in \mathcal{W}^{J}} n_{y, w}^{J} H_{y}^{J,-v}$,

where the $n_{y, w}^{J}$ are such that $n_{w, w}^{J}=1$ and $n_{y, w}^{J} \in v \mathbb{Z}[v]$ if $y \neq w$.

Remark 2.3. In the case $J=\varnothing$, the two parabolic modules coincide with the regular module: $\boldsymbol{M}^{\varnothing}=\boldsymbol{N}^{\varnothing}=\boldsymbol{H}$. Moreover $\underline{H}_{w}^{\varnothing, v^{-1}}=\underline{H}_{w}^{\varnothing,-v}=\underline{H}_{w}$ for all $w \in W$.

From now on, we will focus on the case $u=v^{-1}$; that is, we will deal only with $\boldsymbol{M}^{J}$. The action of the Hecke algebra $\boldsymbol{H}$ on $\boldsymbol{M}^{J}$ is defined as follows. Let $s \in \mathscr{Y}$ be a simple reflection and let $x \in \mathscr{W}^{J}$; then we have (see [Soergel 1997, §3])

$$
\underline{H}_{s} \cdot H_{x}^{J, v^{-1}}= \begin{cases}H_{s x}^{J, v^{-1}}+v H_{x}^{J, v^{-1}} & \text { if } s x \in \mathscr{W}^{J}, s x>x, \\ H_{s x}^{J, v^{-1}}+v^{-1} H_{x}^{J, v^{-1}} & \text { if } s x \in \mathcal{W}^{J}, s x<x, \\ \left(v+v^{-1}\right) H_{x}^{J, v^{-1}} & \text { if } s x \mathbb{W}^{J} .\end{cases}
$$


Definition of the categorification of $\boldsymbol{M}^{\boldsymbol{J}}$. For any category $\mathscr{C}$ which is exact in the sense of [Quillen 1973], let us denote by [C] its Grothendieck group, that is, the abelian group with generators

$$
[X] \text { for } X \in \mathrm{Ob}(\mathscr{b})
$$

and relations

$$
[Y]=[X]+[Z] \quad \text { for every exact sequence } 0 \rightarrow X \rightarrow Y \rightarrow Z \rightarrow 0 .
$$

For an exact endofunctor $F$ on $\mathscr{b}$, denote by $[F]$ the induced endomorphism of [ל].

By a categorification of $\boldsymbol{M}^{J}$, we mean an exact category $\mathscr{C}$ together with an autoequivalence $G$ and a family of exact endofunctors $\left\{F_{s}\right\}_{s \in \mathscr{S}}$ satisfying the following requirements:

(C1) [ $\mathscr{C}]$ becomes an $\mathscr{L}$-module via $v^{i} \cdot[A]=\left[G^{i} A\right]$ for any $i \in \mathbb{Z}$, and there is an isomorphism $h^{J}:[\mathscr{C}] \stackrel{\sim}{\longrightarrow} M^{J}$ of $\mathscr{L}$-modules.

(C2) For any simple reflection $s \in \mathscr{Y}$, we have an isomorphism $G F_{s} \cong F_{s} G$ of functors.

(C3) For any simple reflection $s \in \mathscr{Y}$, the following diagram commutes:

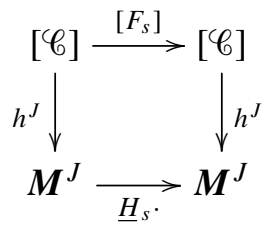

Remark 2.4. Our notion of $\boldsymbol{M}^{J}$-categorification differs from the one in [Mazorchuk and Stroppel 2005, Remark 7.8]. Indeed we made the (weaker) requirement of $\mathscr{b}$ being exact instead of abelian. If we take the above categorification, restrict it to the additive category of projective objects and then abelianise it in the standard way, then this abelianisation is a 2-functor (see [Mazorchuk 2012, §3.3]) and will transform the above categorification into a categorification using abelian categories, in the spirit of [Mazorchuk and Stroppel 2005].

Remark 2.5. Williamson [2011] studied the 2-category of singular Soergel bimodules. A full tensor subcategory of it ( $\varnothing_{\mathscr{B}}^{J}$ in his notation) also provides a categorification of $\boldsymbol{M}^{J}$.

The main goal of this paper is to construct such a categorification. In particular, we will generalise a categorification of the Hecke algebra obtained in [Fiebig 2011], which is known by results in [Fiebig 2008b] to be equivalent to the one via the bimodules of [Soergel 2007]. 


\section{Sheaves on moment graphs}

Definition 3.1 [Fiebig 2008b]. Let $k$ be a field, $V$ a finite-dimensional $k$-vector space, and $\mathbb{P}(V)$ the corresponding projective space. A $V$-moment graph is given by a tuple $(\mathscr{V}, \mathscr{E}, \unlhd, l)$ satisfying these conditions:

$($ MG1) $(\mathscr{V}, \mathscr{E})$ is a graph with a set of vertices $\mathscr{V}$ and a set of edges $\mathscr{E}$.

(MG2) $\unlhd$ is a partial order on $\mathscr{V}$ such that $x, y \in \mathscr{V}$ are comparable if they are linked by an edge.

(MG3) $l: \mathscr{E} \rightarrow \mathbb{P}(V)$ is a map, called the label function.

Remark 3.2. This is the traditional definition. We note that the fact that $\mathscr{V}$ is equipped with a partial order (similarly to the notion of quasihereditary algebra) is used only in the definition of Braden-MacPherson sheaves.

As in [Fiebig 2008b], we think of the order as giving each edge a direction: we write $E: x \rightarrow y \in \mathscr{E}$ if $x \leq y$. We write $x-y$ or $y-x$ if we want to ignore the order.

Bruhat graphs. Let $(\mathcal{W}, \mathscr{Y})$ be a Coxeter system and denote by $m_{s t}$ the order of the product of two simple reflections $s, t \in \mathscr{Y}$. Let $V$ be the geometric representation of $(\mathscr{W}, \mathscr{S})$ (see [Humphreys 1990, §5.3]). Then $V$ is a real vector space with basis indexed by the set of simple reflections $\Pi=\left\{\alpha_{s}\right\}_{s \in \mathscr{S}}$ and $s$ acts on $V$ by

$$
s: v \mapsto v-2\left\langle v, \alpha_{s}\right\rangle \alpha_{s},
$$

where $\langle\cdot, \cdot\rangle: V \times V \longrightarrow \mathbb{R}$ is the symmetric bilinear form given by

$$
\left\langle\alpha_{s}, \alpha_{t}\right\rangle= \begin{cases}-\cos \left(\pi / m_{s t}\right) & \text { if } m_{s t} \neq \infty \\ -1 & \text { if } m_{s t}=\infty\end{cases}
$$

Consider a subset $J \subseteq \mathscr{Y}$ and keep the same notation as in the previous section. Choose $\lambda \in V$ such that $\mathscr{W}_{J}=\operatorname{Stab}_{W}(\lambda)$. Then $\mathscr{W}^{J}$ can be identified with the orbit $W \cdot \lambda$ via $x \mapsto x(\lambda)$.

Recall that the set of reflections $\mathscr{T}$ of $\mathscr{W}$ is

$$
\mathscr{T}=\left\{w s w^{-1} \mid s \in \mathscr{S}, w \in \mathcal{W}\right\} .
$$

Definition 3.3 [Fiebig 2008b, §2.2]. The Bruhat moment graph $\varphi^{J}$ associated to the Coxeter datum $(\mathcal{W}, \mathscr{Y}, J)$ is the following $V$-moment graph:

- The set of vertices is given by $\mathscr{W} \cdot \lambda \leftrightarrow W^{J}$, and $x \rightarrow y$ is an edge if and only if $\ell(x)<\ell(y)$ and there exists a reflection $t \in \mathcal{T}$ such that $x(\lambda)=t y(\lambda)$, that is, $y=t x w$ for some $w \in W_{J}$ and $y \notin x W_{J}$.

- The partial order $W^{J}$ is the (induced) Bruhat order.

- $l(x \rightarrow t x w)$ is given by the line generated by $x(\lambda)-t x(\lambda)$ in $\mathbb{P}(V)$. 
Consider now two Bruhat moment graphs on $V$, namely $\mathscr{G}=\mathscr{G}(\mathcal{W}, \mathscr{Y}, \varnothing)$ and $\mathscr{G}^{J}=\mathscr{G}(\mathscr{W}, \mathscr{Y}, J)$. The canonical quotient map $p^{J}: \mathscr{G} \rightarrow \mathscr{G}^{J}$ is induced by the map $p_{\mathscr{V}}^{J}: x \rightarrow x^{J}$, with $x^{J}$ a minimal length representative of the coset $x^{\mathscr{Q}} W_{J}$.

Example 3.4. Let $\mathscr{W}=S_{3}$, the symmetric group on three letters. In this case we have $V=\mathbb{R}^{2}, \Pi=\{\alpha, \beta\}$, and the angle between the two roots is $2 \pi / 3$. If we fix $J=\left\{s_{\alpha}\right\}$, then $p^{J}$ is as follows.
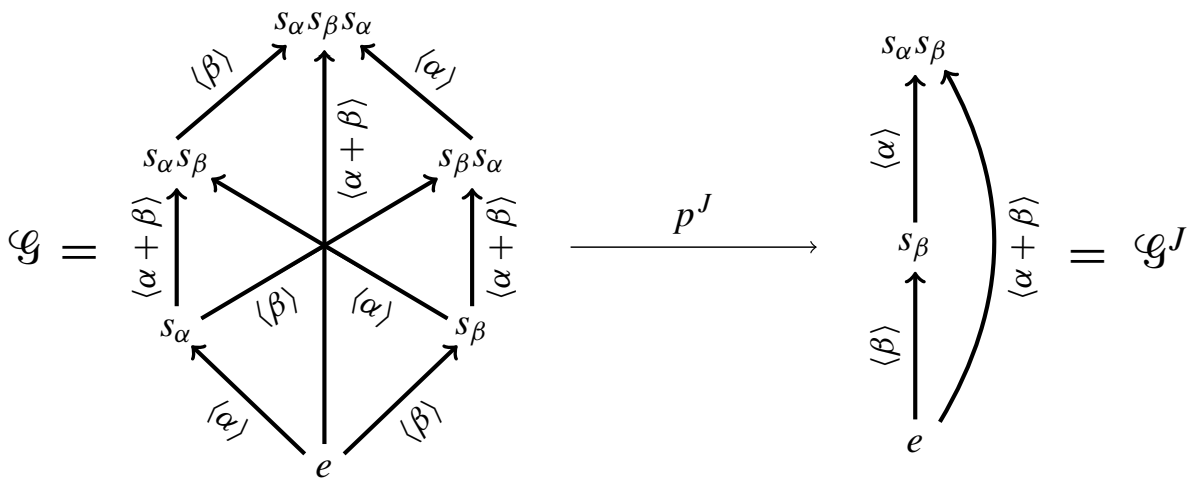

We have

$$
\begin{aligned}
p_{\mathscr{V}}^{J}(e) & =p_{\mathscr{V}}^{J}\left(s_{\alpha}\right)=e, \\
p_{\mathscr{V}}^{J}\left(s_{\beta}\right) & =p_{\mathscr{V}}^{J}\left(s_{\beta} s_{\alpha}\right)=s_{\beta}, \\
p_{\mathscr{V}}^{J}\left(s_{\alpha} s_{\beta}\right) & =p_{\mathscr{V}}^{J}\left(s_{\alpha} s_{\beta} s_{\alpha}\right)=s_{\alpha} s_{\beta} .
\end{aligned}
$$

\section{Sheaves on a $V$-moment graph.}

Conventions. For any finite-dimensional vector space $V$ over the field $k$ (with char $k \neq 2$ ), we denote by $S=\operatorname{Sym}(V)$ its symmetric algebra. Then $S$ is a polynomial ring and we provide it with the grading induced by setting $S_{\{2\}}=V$. From now on, all the $S$-modules will be finitely generated and $\mathbb{Z}$-graded. Moreover we will consider only degree-zero morphisms between them. For a graded $S$-module $M=\bigoplus_{i} M_{\{i\}}$ and for $j \in \mathbb{Z}$, we denote by $M\langle j\rangle$ the $\mathbb{Z}$-graded $S$-module obtained from $M$ by shifting the grading by $j$, that is, $(M\langle j\rangle)_{\{i\}}=M_{\{j+i\}}$.

Definition 3.5 [Braden and MacPherson 2001]. Let $\mathscr{G}=(\mathscr{V}, \mathscr{E}, \unlhd, l)$ be a $V$-moment graph. A sheaf $\mathscr{F}_{\mathcal{F}}$ on $\mathscr{G}$ consists of $\left(\left\{\mathscr{F}^{x}\right\},\left\{\mathscr{F}^{E}\right\},\left\{\rho_{x, E}\right\}\right)$ satisfying these conditions:

(SH1) $\mathscr{F}^{x}$ is an $S$-module for all $x \in \mathscr{V}$.

(SH2) $\mathscr{F}^{E}$ is an $S$-module such that $l(E) \cdot \mathscr{F}^{E}=\{0\}$ for all $E \in \mathscr{E}$.

(SH3) $\rho_{x, E}: \mathscr{F}^{x} \rightarrow \mathscr{F}^{E}$ is a homomorphism of $S$-modules, for all $x \in \mathscr{V}, E \in \mathscr{E}$ with $x$ incident to the edge $E$. 
Remark 3.6. We may consider the following topology on the space $\Gamma=\mathscr{V} \cup \mathscr{E}$ (see [Braden and MacPherson 2001, §1.3]). We say that a subset $O \subseteq \Gamma$ is open if whenever a vertex $x$ is in $O$, then all edges adjacent to $x$ are also in $O$. With this topology, the object in Definition 3.5 is a sheaf of $S$-modules on $\Gamma$ in the usual sense. For our purposes, it will be sufficient to consider sheaves as purely combinatorial, algebraic objects.

Example 3.7 [Braden and MacPherson 2001, §1]. The structure sheaf $\mathscr{E}$ of $V$ moment graph $\mathscr{G}=(\mathscr{V}, \mathscr{E}, \unlhd, l)$ is defined as follows:

- $\mathscr{L}^{x}=S$ for all $x \in \mathscr{V}$.

- $\mathscr{L}^{E}=S / l(E) \cdot S$ for all $E \in \mathscr{E}$.

- $\rho_{x, E}: S \rightarrow S / l(E) \cdot S$ is the canonical quotient map, for all $x \in \mathscr{V}$ and $E \in \mathscr{E}$ such that $x$ is incident to the edge $E$.

Definition 3.8 [Fiebig 2009]. Let $\mathscr{G}=(\mathscr{V}, \mathscr{E}, \unlhd, l)$ be a $V$-moment graph and let $\mathscr{F}=\left(\left\{\mathscr{F}^{x}\right\},\left\{\mathscr{F}^{E}\right\},\left\{\rho_{x, E}\right\}\right), \mathscr{F}^{\prime}=\left(\left\{\mathscr{F}^{\prime x}\right\},\left\{\mathscr{F}^{\prime} E\right\},\left\{\rho_{x, E}^{\prime}\right\}\right)$ be two sheaves on $\mathscr{G}$. A morphism $\varphi: \mathscr{F} \longrightarrow \mathscr{F}^{\prime}$ is given by the following data:

(MSH1) $\varphi^{x}: \mathscr{F}^{x} \rightarrow \mathscr{F}^{\prime x}$ is a homomorphism of $S$-modules, for all $x \in \mathscr{V}$.

(MSH2) $\varphi^{E}: \mathscr{F}^{E} \rightarrow \mathscr{F}^{E}$ is a homomorphism of $S$-modules, for all $E \in \mathscr{E}$, and if $x \in \mathscr{V}$ is incident to the edge $E$, the following diagram commutes:

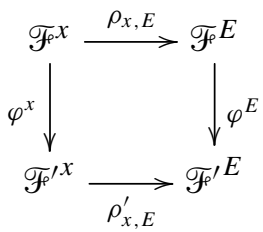

Definition 3.9. Let $G$ be a $V$-moment graph. We denote by $\mathbf{S h}(\varphi)$ the category of sheaves on $\mathscr{G}$ and corresponding morphisms.

Remark 3.10. The category of sheaves on $\varphi$ is graded, with the shift of grading autoequivalence $\langle 1\rangle: \mathbf{S h}(\mathscr{G}) \rightarrow \mathbf{S h}(\mathscr{G})$ given by

$$
\left(\left\{\mathscr{F}^{x}\right\},\left\{\mathscr{F}^{E}\right\},\left\{\rho_{x, E}\right\}\right) \mapsto\left(\left\{\mathscr{F}^{x}\langle 1\rangle\right\},\left\{\mathscr{F}^{E}\langle 1\rangle\right\},\left\{\rho_{x, E} \circ\langle 1\rangle\right\}\right) .
$$

Moreover $\mathbf{S h}(\varphi)$ is an additive category, with zero object $(\{0\},\{0\},\{0\})$, biproduct given by

$$
\begin{aligned}
\left(\left\{\mathscr{F}^{x}\right\},\left\{\mathscr{F}^{E}\right\},\left\{\rho_{x, E}\right\}\right) \oplus\left(\left\{\mathscr{F}^{\prime x}\right\},\left\{\mathscr{F}^{\prime}\right\},\right. & \left.\left\{\rho_{x, E}^{\prime}\right\}\right) \\
& =\left(\left\{\mathscr{F}^{x} \oplus \mathscr{F}^{\prime x}\right\},\left\{\mathscr{F}^{E} \oplus \mathscr{F}^{\prime} E\right\},\left\{\left(\rho_{x, E}, \rho_{x, E}^{\prime}\right)\right\}\right),
\end{aligned}
$$

and idempotent split. 
Sections of a sheaf on a moment graph. Even if $\mathbf{S h}(G)$ is not a category of sheaves in the usual sense, we may define the notion of sections following [Fiebig 2008a].

Definition 3.11. Let $\mathscr{G}=(\mathscr{V}, \mathscr{E}, \unlhd, l)$ be a $V$-moment graph,

$$
\mathscr{F}=\left(\left\{\mathscr{F}^{x}\right\},\left\{\mathscr{F}^{E}\right\},\left\{\rho_{x, E}\right\}\right) \in \mathbf{S h}(\mathscr{G}),
$$

and $\mathscr{I} \subseteq \mathscr{V}$. The set of sections of $\mathscr{F}$ over $\mathscr{I}$ is denoted by $\Gamma(\mathscr{F}, \mathscr{F})$ and defined as

$$
\Gamma(\mathscr{I}, \mathscr{F}):=\left\{\left(m_{x}\right) \in \prod_{x \in \mathscr{I}} \mathscr{F}^{x} \mid \begin{array}{c}
\rho_{x, E}\left(m_{x}\right)=\rho_{y, E}\left(m_{y}\right) \\
\text { for all } E: x-y \in \mathscr{E}, x, y \in \mathscr{I}
\end{array}\right\} .
$$

We denote by $\Gamma(\mathscr{F}):=\Gamma(\mathscr{V}, \mathscr{F})$ the set of global sections of $\mathscr{F}$.

Example 3.12. A very important example is given by the set of global sections of the structure sheaf (see Example 3.7). In this case, we get the structure algebra:

$$
\mathscr{L}:=\Gamma(\mathscr{L})=\left\{\left(z_{x}\right)_{x \in \mathscr{V}} \in \prod_{x \in \mathscr{V}} S \mid \begin{array}{c}
z_{x}-z_{y} \in l(E) \cdot S \\
\text { for all } E: x-y \in \mathscr{E}
\end{array}\right\} .
$$

Remark 3.13. The algebra $\mathscr{E}$ should be thought of as the centre of a noncritical block in the deformed category 0 (see [Fiebig 2003, Theorem 3.6]).

It is easy to check that $\mathscr{L}$, equipped with componentwise addition and multiplication, is an algebra and that there is an action of $S$ on it by diagonal multiplication. Moreover for any sheaf $\mathscr{F} \in \mathbf{S h}(\mathscr{G})$, the structure algebra $\mathscr{E}$ acts on the space $\Gamma(\mathscr{F})$ via componentwise multiplication, so $\Gamma$ defines a functor from the category of sheaves on $\mathscr{G}$ to the category of $\mathscr{L}$-modules:

$$
\Gamma: \mathbf{S h}(\mathscr{G}) \rightarrow \mathscr{L}-\bmod .
$$

BMP-sheaves. Let $\mathscr{G}=(\mathscr{V}, \mathscr{E}, \unlhd, l)$ be a $V$-moment graph. For all $\mathscr{F} \in \mathbf{S h}(\mathscr{G})$ and $x \in \mathscr{V}$, we set

$$
\begin{aligned}
& \mathscr{E}_{\delta x}:=\{E \in \mathscr{E} \mid \text { there is } y \in \mathscr{V} \text { with } E: x \rightarrow y\}, \\
& \mathscr{V}_{\delta x}:=\left\{y \in \mathscr{V} \mid \text { there is } E \in \mathscr{E}_{\delta x} \text { with } E: x \rightarrow y\right\} .
\end{aligned}
$$

Additionally for any $x \in \mathscr{V}$ denote $\{\triangleright x\}=\{y \in \mathscr{V} \mid y \triangleright x\}$ and define $\mathscr{F}^{\delta} \delta$ as the image of $\Gamma(\{\triangleright x\}, \mathscr{F})$ under the composition of the following functions:

$$
u_{x}: \Gamma(\{\triangleright x\}, \mathscr{F}) \longrightarrow \bigoplus_{y \triangleright x} \mathscr{F}^{y} \longrightarrow \bigoplus_{y \in \mathcal{V}_{\delta x}} \mathscr{F}_{\mathcal{F}} y \stackrel{\bigoplus \rho_{y, E}}{\longrightarrow} \bigoplus_{E \in \in_{\delta}^{\mathscr{6} \delta x}} \mathscr{F}^{E}
$$

Theorem 3.14 [Braden and MacPherson 2001]. Suppose $\mathscr{G}=(\mathscr{V}, \mathscr{E}, \unlhd, l)$ is a $V$-moment graph and let $w \in \mathscr{V}$. There exists a unique up to isomorphism indecomposable sheaf $\mathscr{B}(w)$ on $\mathscr{G}$ with the following properties: 
(BMP1) If $x \in \mathscr{V}$, then $\mathscr{B}(w)^{x} \cong 0$, unless $x \unlhd w$. Moreover $\mathscr{B}(w)^{w} \cong S$.

(BMP2) If $x, y \in \mathscr{V}$ and $E: x \rightarrow y \in \mathscr{E}$, then the map $\rho_{y, E}: \mathscr{B}(w)^{y} \rightarrow \mathscr{B}(w)^{E}$ is surjective, with kernel $l(E) \cdot \mathscr{B}(w)^{y}$.

(BMP3) If $x, y, w \in \mathscr{V}, x \neq w$ and $E: x \rightarrow y \in E$, then $\rho_{\delta x}:=\bigoplus_{E \in \mathscr{E}_{\delta x}} \rho_{x, E}:$ $\mathscr{B}(w)^{x} \rightarrow \mathscr{B}(w)^{\delta x}$ is a projective cover in the category of graded $S$ modules.

We call $\mathscr{B}(w)$ the $B M P$ sheaf.

\section{Modules over the structure algebra}

Let $\mathscr{E}$ be the structure algebra (see p. 424) of a regular Bruhat graph $\mathscr{G}=\mathscr{G}(\mathcal{W}, \varnothing)$ and denote by $\mathscr{L}$-mod ${ }^{\mathrm{f}}$ the category of $\mathbb{Z}$-graded $\mathscr{L}$-modules that are torsion-free and finitely generated over $S$. Fiebig [2008b] defined translation functors on the category $\mathscr{L}$-mod ${ }^{\mathrm{f}}$. Using these, he defined inductively a full subcategory $\mathscr{H}$ of $\mathscr{L}$-mod and proved that $\mathscr{H}$, in characteristic zero, is equivalent to a category of bimodules introduced by Soergel [2007]. In [Fiebig 2011], it is shown that $\mathscr{H}$ categorifies the Hecke algebra $\boldsymbol{H}$ (and the periodic module $\boldsymbol{M}$ ), using translation functors. The aim of this section is to define translation functors in the parabolic setting and to extend some results of [Fiebig 2011].

Let $\mathscr{W}$ be a Weyl group, let $\mathscr{Y}$ be its set of simple reflections and let $J \subseteq \mathscr{Y}$. Hereafter we will keep the notation we used in Section 2. Recall that for any $z \in \mathcal{W}$, there is a unique factorisation $x=x^{J} x_{J}$, with $x^{J} \in \mathscr{W}^{J}, x_{J} \in \mathcal{W}_{J}$ and $\ell(x)=\ell\left(x^{J}\right)+\ell\left(x_{J}\right)$ (see [Björner and Brenti 2005, Proposition 2.4.4]).

In [Fiebig 2008b], for all $s \in \mathscr{Y}$, an involutive automorphism $\sigma_{s}$ of the structure algebra of a regular Bruhat graph is given. In a similar way, we will define an involution ${ }_{s} \sigma$ for a fixed simple reflection $s \in \mathscr{Y}$ on the structure algebra $\mathscr{L}^{J}$ of the parabolic Bruhat moment graph $\varphi^{J}$.

Let $x, y \in W^{J}$. Notice that $l(x-y)=\alpha_{t}$ if and only if $l(s x-s y)=s\left(\alpha_{t}\right)$ because $s x w(s y)^{-1}=s x w y^{-1} s=s t s$, for some $w \in W_{J}$.

Denote by $\tau_{s}$ the automorphism of the symmetric algebra $S$ induced by the mapping $\lambda \mapsto s(\lambda)$ for all $\lambda \in V$. For $\left(z_{x}\right)_{x \in W^{J}} \in \mathscr{L}^{J}$, we set ${ }_{s} \sigma\left(\left(z_{x}\right)_{x \in W^{J}}\right)=$ $\left(z_{x}^{\prime}\right)_{x \in W^{J}}$, where $z_{x}^{\prime}:=\tau_{s}\left(z_{(s x)^{J}}\right)$. This is again an element of the structure algebra from what we have observed above.

Let us fix the following notation:

- $s \mathscr{L}^{J}$ denotes the space of invariants with respect to ${ }_{s} \sigma$;

- ${ }^{-s \mathscr{L} J}$ denotes the space of anti-invariants with respect to ${ }_{s} \sigma$.

We denote by $\bar{\alpha}_{s}$ the element of $\mathscr{L}^{J}$ whose components are all equal to $\alpha_{s}$. We obtain the following decomposition of $\mathscr{L}^{J}$ as a ${ }^{s \mathscr{L}} \mathscr{L}^{J}$-module:

Lemma 4.1.

$$
\mathscr{L}^{J}={ }^{s} \mathscr{L}^{J} \oplus \bar{\alpha}_{s} \cdot{ }^{\prime} \mathscr{L}^{J} .
$$


Proof. Because ${ }_{s} \sigma$ is an involution, we get $\mathscr{L}^{J}={ }^{s} \mathscr{L}^{J} \oplus \oplus^{-s \mathscr{L}} \mathscr{L}^{J}$. Since $\bar{\alpha}_{s} \in \mathscr{L}^{J}$ and $s\left(\alpha_{s}\right)=-\alpha_{s}$, it follows that ${ }_{s} \sigma\left(\bar{\alpha}_{s}\right)=-\bar{\alpha}_{s}$ and so $\bar{\alpha}_{s} \cdot{ }^{s \mathscr{L} J} \subseteq{ }^{-s \mathscr{L} J}$ and we now have to prove the other inclusion, that is, every element $z \in \in^{-s \mathscr{Z} J}$ is divisible by $\bar{\alpha}_{s}$ in $-s \mathscr{E} J$.

If $z=\left(z_{x}\right) \in^{-s \mathscr{E}^{J}}$, then for all $x \in \mathscr{W}^{J}$,

$$
z_{x}=-\tau_{s}\left(z_{(s x)^{J}}\right) \equiv-z_{(s x)^{J}} \quad\left(\bmod \alpha_{s}\right) .
$$

On the other hand,

$$
z_{x} \equiv z_{(s x)^{J}} \quad\left(\bmod \alpha_{s}\right)
$$

It follows that $2 z_{x} \equiv 0\left(\bmod \alpha_{s}\right)$, that is, $\alpha_{s}$ divides $z_{x}$ in $S$.

It remains to verify that $z^{\prime}:=\bar{\alpha}_{s}{ }^{-1} \cdot z \in \mathscr{L}$, that is, $z_{x}^{\prime}-z_{(t x)^{J}}^{\prime} \equiv 0\left(\bmod \alpha_{t}\right)$ for any $x \in \mathscr{W}^{J}$ and $t \in \mathscr{T}$. If $(t x)^{J}=(s x)^{J}$ there is nothing to prove since $\alpha_{s}$ divides $z_{x}^{\prime}=z_{(s x)^{J}}$ and $z_{(s x)^{J}}^{\prime}=z_{x}$ and hence also their difference. On the other hand, if $(t x)^{J} \neq(s x)^{J}$ we get the following:

$$
\alpha_{s} \cdot\left(z_{x}^{\prime}-z_{(t x)^{J}}^{\prime}\right)=z_{x}-z_{(t x)^{J}} \equiv 0\left(\bmod \alpha_{t}\right) .
$$

Since $\alpha_{s}$ and $\alpha_{t}$ are linearly independent, $\alpha_{s} \not \equiv 0\left(\bmod \alpha_{t}\right)$ and we obtain

$$
z_{x}^{\prime}-z_{(t x)^{J}}^{\prime} \equiv 0\left(\bmod \alpha_{t}\right)
$$

Translation functors and special modules. In order to define translation functors, we need an action of $S$ on ${ }^{s \mathscr{L}} J$ and $\mathscr{L}^{J}$.

Lemma 4.2. For any $\lambda \in V$ and any $x \in \mathcal{W}^{J}$, let us set

$$
c(\lambda)_{x}^{J}:=\sum_{x_{J} \in \mathscr{W}_{J}} x x_{J}(\lambda)
$$

Then $c(\lambda)^{J}:=\left(c(\lambda)_{x}^{J}\right)_{x \in \mathscr{W}^{J}} \in{ }^{\operatorname{seL}}{ }^{J}$.

Proof. First we prove that $c(\lambda)^{J} \in \mathscr{L}^{J}$, that is, $c(\lambda)_{x}^{J}-c(\lambda)_{(t x)^{J}}^{J} \equiv 0\left(\bmod \alpha_{t}\right)$. Since for any $x_{J}$ there exists an element $y_{J}$ such that $x x_{J}=t(t x)^{J} y_{J}$, we obtain

$$
\begin{aligned}
\sum_{x_{J} \in \mathscr{W}_{J}} x x_{J}(\lambda)-\sum_{x_{J} \in \mathscr{W}_{J}}(t x)^{J} x_{J}(\lambda) & =\sum_{y_{J} \in \mathscr{W}_{J}} t(t x)^{J} y_{J}(\lambda)-\sum_{y_{J} \in \mathscr{W}_{J}}(t x)^{J} y_{J}(\lambda) \\
& =t\left(\sum_{y_{J} \in \mathscr{W}_{J}}(t x)^{J} y_{J}(\lambda)\right)-\sum_{y_{J} \in \mathscr{W}_{J}}(t x)^{J} y_{J}(\lambda) \\
& =\left(\sum_{y_{J} \in \mathscr{W}_{J}} 2\left\langle(t x)^{J} y_{J}(\lambda), \alpha_{t}\right\rangle\right) \alpha_{t} \\
& \equiv 0\left(\bmod \alpha_{t}\right) .
\end{aligned}
$$


To conclude it is left to show that $c(\lambda)^{J}$ is invariant with respect to ${ }_{s} \sigma$. For any $x \in \mathcal{W}^{J}$, one has

$$
\tau_{s}\left(c(\lambda)_{x}^{J}\right)=\tau_{s}\left(\sum_{x_{J} \in \mathscr{W}_{J}} x x_{J}(\lambda)\right)=\sum_{x_{J} \in \mathscr{W}_{J}} s x x_{J}(\lambda)=c(\lambda)_{s x}^{J} .
$$

Hence we have ${ }_{s} \sigma\left(c(\lambda)^{J}\right)=\left(\tau_{s} c(\lambda)_{s x}^{J}\right)_{x \in W^{J}}=c(\lambda)^{J}$.

For any $x \in \mathscr{W}^{J}$, denote by $\eta_{x}$ the map of (free) $S^{W_{J}}$-modules $S \rightarrow S^{W_{J}}$ induced by the map $\lambda \mapsto c(\lambda)_{x}^{J}$ for all $\lambda \in V$. Now by Lemma 4.2, the action of $S$ on $\mathscr{L}^{J}$ given by

$$
p .\left(z_{x}\right)_{x \in W^{J}}=\left(\eta_{x}(p) z_{x}\right), \quad p \in S, \quad z \in \mathscr{L}^{J},
$$

preserves ${ }^{s \mathscr{L}} \mathscr{L}^{J}$. Thus any $\mathscr{L}^{J}$-module or ${ }^{S \mathscr{L}} \mathscr{L}^{J}$-module has an $S$-module structure as well. Suppose $\mathscr{L}^{J}$-mod ${ }^{\mathrm{f}}$, respectively, ${ }^{s} \mathscr{L}$ - $\bmod ^{\mathrm{f}}$, is the category of $\mathbb{Z}$-graded $\mathscr{L}^{J}$-modules, respectively, ${ }^{s} \mathscr{L}^{J}$-modules, that are torsion-free and finitely generated over $S$, respectively, $S^{W_{J}}$-modules.

The translation on the wall is the functor ${ }^{s, \mathrm{on}} \theta: \mathscr{L}^{J}-\bmod \rightarrow{ }^{s \mathscr{L}}{ }^{J}$-mod defined

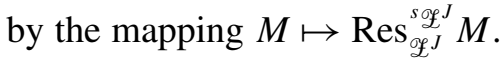

The translation out of the wall is the functor ${ }^{s, \text { out }} \theta:{ }^{s \mathscr{L}}{ }^{J}-\bmod \rightarrow \mathscr{L}^{J}$-mod defined by the mapping $N \mapsto \operatorname{Ind}_{\mathscr{L} J}^{\text {sóJ } J} N=\mathscr{L}^{J} \otimes_{\text {só } J} N$. Observe that this functor is well defined due to Lemma 4.1.

By composition, we get a functor ${ }^{s} \theta^{J}:={ }^{s, \text { out }} \theta \circ^{s, \text { on }} \theta: \mathscr{L}^{J}$-mod $\rightarrow \mathscr{L}^{J}$-mod that we call the (left) translation functor.

Remark 4.3. This construction is very similar to the one in [Soergel 1990], where translation functors are defined in the finite case for the coinvariant algebra.

Remark 4.4. One could consider the idempotent split additive tensor category generated by the translation functors we defined above and describe indecomposable projective objects. This would be useful in order to strengthen our main result to a proper categorification (see Remark 5.9). In this paper we are not going to investigate this category of translation functors but the one of special modules, defined on p. 428.

The following proposition describes the first properties of ${ }^{s} \theta$ :

Proposition 4.5. (1) The functors from ${ }^{s \mathscr{L} J}$-mod to $\mathscr{L}^{J}$-mod mapping $M$ to $\mathscr{L}^{J}\langle 2\rangle \otimes_{\text {só } J} M$ and $\operatorname{Hom}_{s \mathscr{L} J}\left(\mathscr{L}^{J}, M\right)$, respectively, are isomorphic.

(2) The functor ${ }^{s} \theta=\mathscr{L}^{J} \otimes_{\text {só } J}-: \mathscr{L}^{J}$-mod $\rightarrow \mathscr{L}^{J}$-mod is self-adjoint up to a shift. 


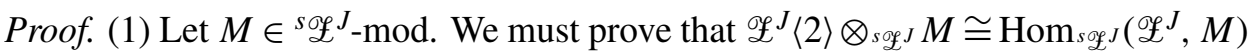
as $\mathscr{L}^{J}$-modules.

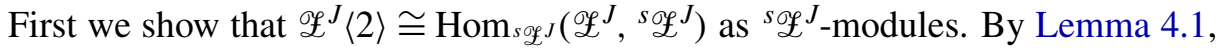

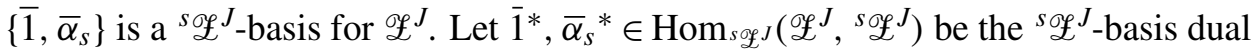
to $\overline{1}$ and $\bar{\alpha}_{s}$, that is,

$$
\overline{1}^{*}(\overline{1})=\overline{1}, \quad \overline{1}^{*}\left(\bar{\alpha}_{s}\right)=\overline{0}, \quad \bar{\alpha}_{s}^{*}\left(\bar{\alpha}_{s}\right)=\overline{1}, \quad \bar{\alpha}_{s}^{*}(\overline{1})=\overline{0},
$$

where $\overline{1} \in{ }^{s \mathscr{W}} J$, respectively, $\overline{0} \in{ }^{s \mathscr{Z}}{ }^{J}$, is the section with 1 , respectively, 0 , in all entries. Since $\operatorname{deg}(1)-2=-2=\operatorname{deg}\left(\bar{\alpha}_{s}{ }^{*}\right)$ and $\operatorname{deg}\left(\bar{\alpha}_{s}\right)-2=0=\operatorname{deg} \overline{1}^{*}$, we

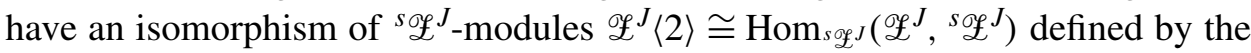
mapping

$$
\overline{1} \mapsto \bar{\alpha}_{s}^{*}, \quad \bar{\alpha}_{s} \mapsto \overline{1}^{*} .
$$

Because $\mathscr{L}^{J}$ is free of rank two over ${ }^{\mathscr{L}} \mathscr{E}^{J}$,

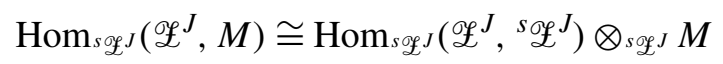

by the map

$$
\varphi \mapsto \bar{\alpha}_{s}^{*} \otimes \varphi\left(\bar{\alpha}_{s}\right)+\overline{1}^{*} \otimes \varphi(\overline{1}) .
$$

This conclude the proof of (1).

(2) Since $\mathscr{L}^{J} \otimes_{s_{\mathscr{L}} J}-$ and $\operatorname{Hom}_{s \mathscr{L} J}\left(\mathscr{L}^{J},-\right)$ are, respectively, left- and right-adjoint to the restriction functor, we obtain the following chain of isomorphisms for any pair $M, N \in \mathscr{L}^{J}$ :

$$
\begin{aligned}
& \operatorname{Hom}_{\mathscr{L} J}\left({ }^{s} \theta M, N\right)=\operatorname{Hom}_{\mathscr{L} J}\left(\mathscr{L}^{J} \otimes \mathscr{s \mathscr { L } J}\left(\operatorname{Re}_{\mathscr{L}_{\mathscr{L} J} J}^{s_{\mathscr{L}} J} M\right), N\right)
\end{aligned}
$$

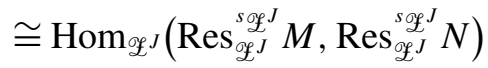

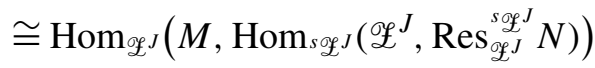

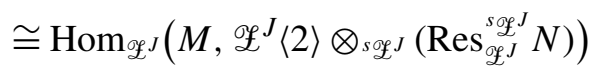

$$
\begin{aligned}
& =\operatorname{Hom}_{\mathscr{L} J}\left(M,{ }^{s} \theta\langle 2\rangle N\right) \text {. }
\end{aligned}
$$

Parabolic special modules. As in [Fiebig 2008b], we define inductively a full subcategory of $\mathscr{L}^{J}$-mod.

Let $B_{e}^{J} \in \mathscr{L}^{J}$-mod be the free $S$-module of rank one on which $z=\left(z_{x}\right)_{x \in \mathscr{W}^{J}}$ acts via multiplication by $z_{e}$.

Definition 4.6. The category $\mathscr{H}^{J}$ of special $\mathscr{L}^{J}$-modules is the full subcategory of $\mathscr{L}^{J}-$ mod $^{\mathrm{f}}$ whose objects are isomorphic to a direct summand of a direct sum of modules of the form ${ }^{s_{i_{1}}} \theta \circ \ldots \circ{ }^{s_{i_{r}}} \theta\left(B_{e}^{J}\right)\langle n\rangle$, where $s_{i_{1}}, \ldots, s_{i_{r}} \in \mathscr{Y}$ and $n \in \mathbb{Z}$.

The category $s \mathscr{H}^{J}$ of special ${ }^{s \mathscr{L}} \mathscr{L}^{J}$-modules is the full subcategory of ${ }^{s} \mathscr{L}^{J}$-mod ${ }^{\mathrm{f}}$ whose objects are isomorphic to a direct summand of ${ }^{s, \text { on }} \theta(M)$ for some $M \in \mathscr{H}^{J}$. 
Finiteness of special modules. Let $\Omega$ be a finite subset of $\mathscr{W}^{J}$ and denote by $\mathscr{L}^{J}(\Omega)$ the sections of the structure sheaf over $\Omega$, that is,

$$
\mathscr{L}^{J}(\Omega)=\left\{\left(z_{x}\right) \in \prod_{x \in \Omega} S \mid \begin{array}{c}
z_{x} \equiv z_{y}\left(\bmod \alpha_{t}\right) \\
\text { if there is } w \in \mathscr{W}_{J} \text { s.t. } y w x^{-1}=t \in \mathcal{T}
\end{array}\right\} .
$$

If $\Omega \subseteq W^{J}$ is $s$-invariant, that is, $s \Omega=\Omega$, we may restrict ${ }_{s} \sigma$ to it. We denote by $\mathscr{S}^{J}(\Omega) \subseteq \mathscr{L}^{J}(\Omega)$ the space of invariants and using Lemma 4.1, we get a decomposition $\mathscr{L}^{J}(\Omega)={ }^{s} \mathscr{L}^{J}(\Omega) \oplus \bar{\alpha}_{s} \cdot{ }^{\operatorname{s} \mathscr{L}} J(\Omega)$.

In the following lemma we prove the finiteness of special $\mathscr{L}^{J}$-modules as Fiebig [2011] does for special $\mathscr{L}$-modules.

Lemma 4.7. (1) Let $M \in \mathscr{H}^{J}$. Then there exists a finite subset $\Omega \subset \mathcal{W}^{J}$ such that $\mathscr{L}^{J}$ acts on $M$ via the canonical map $\mathscr{L}^{J} \rightarrow \mathscr{L}^{J}(\Omega)$.

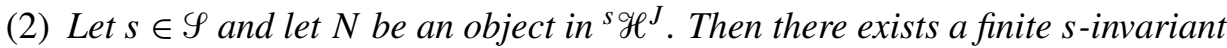
subset $\Omega \subset \mathscr{W}^{J}$ such that ${ }^{s \mathscr{L} J}$ acts on $N$ via the canonical map ${ }^{\operatorname{soL}} J \rightarrow{ }^{s \mathscr{L}} J(\Omega)$.

Proof. We prove (1) by induction. It holds clearly for $B_{e}$, since $\mathscr{L}^{J}$ acts on it via the map $\mathscr{L}^{J} \rightarrow \mathscr{L}^{J}(\{e\})$. Now we have to show that if the claim is true for $M \in \mathscr{H}^{J}$, then it holds also for ${ }^{s} \theta(M)$. Suppose $\mathscr{L}^{J}$ acts via the map $\mathscr{L}^{J} \rightarrow \mathscr{L}^{J}(\Omega)$ over $M$. Observe that we may assume $\Omega s$-invariant since we can just replace it by $\Omega \cup s \Omega$, which is still finite. In this way the ${ }^{s \mathscr{L}} \mathscr{L}^{J}$-action on ${ }^{s} \theta M$ factors via ${ }^{s} \mathscr{L}^{J} \rightarrow{ }^{s \mathscr{L}} J(\Omega)$ and so we obtain ${ }^{s} \theta M:=\mathscr{L}^{J} \otimes_{\text {só } J} M=\mathscr{L}^{J}(\Omega) \otimes_{\text {só }^{J}(\Omega)} M$.

Claim (2) follows directly from claim (1).

\section{Modules with Verma flag and statement of the main result}

We recall some notation from [Fiebig 2008a]. Let $Q$ be the quotient field of $S$ and let $A$ be an $S$-module. Then we denote by $A_{Q}=A \otimes_{S} Q$. Let us assume $\mathscr{G}$ to be such that for any $M \in \mathscr{L}$-mod ${ }^{\mathrm{f}}$ there is a canonical decomposition

$$
M_{Q}=\bigoplus_{x \in \mathcal{V}} M_{Q}^{x}
$$

and so a canonical inclusion $M \subseteq \bigoplus_{\mathcal{Q}} M_{Q}^{x}$. For all subsets of the set of vertices $\Omega \subseteq \mathscr{V}$, we may define

$$
\begin{aligned}
& M_{\Omega}:=M \cap \bigoplus_{x \in \Omega} M_{Q}^{x}, \\
& M^{\Omega}:=M / M_{\mathscr{V} \backslash \Omega}=\operatorname{im}\left(M \rightarrow M_{Q} \rightarrow \bigoplus_{x \in \Omega} M_{Q}^{x}\right) .
\end{aligned}
$$

For all $x \in \mathscr{V}$, we set

$$
M_{[x]}:=\operatorname{ker}\left(M^{\{\unrhd x\}} \rightarrow M^{\{\triangleright x\}}\right)
$$


and if $x \triangleleft y$ and $[x, y]=\{x, y\}$, we denote

$$
M_{[x, y]}:=\operatorname{ker}\left(M^{\{\unrhd x\}} \rightarrow M^{\{\unrhd x\} \backslash\{x, y\}}\right) .
$$

Remark 5.1. In [Fiebig 2008a], the module $M_{[x]}$ is denoted by $M^{[x]}$. The notation we are adopting in this paper is the one from [Fiebig 2011].

Modules with a Verma flag. From now on, let $\mathscr{G}$ be a Bruhat moment graph. In [Fiebig 2008a] it is shown that in this case any $M \in \mathscr{L}$-mod ${ }^{\mathrm{f}}$ admits a decomposition like (7) and hence the modules $M_{[x]}$ are well defined for any $x \in \mathscr{V}$.

Let $\mathscr{V}$ denote the full subcategory of $\mathscr{L}$-mod ${ }^{\mathrm{f}}$ whose objects admit a Verma flag, that is, $M \in \mathscr{V}$ if and only if $M^{\Omega}$ is a graded free $S$-module for any $\Omega \subseteq \mathscr{V}$ upwardly closed with respect to the partial order in the set of vertices. In our hypotheses this condition is equivalent to $M_{[x]}$ being a graded free $S$-module for any $x \in \mathscr{V}$ (see [Fiebig 2008a, Lemma 4.7]).

Exact structure. Now we want to equip the category $\mathcal{V}$ with an exact structure.

Definition 5.2. A sequence $L \rightarrow M \rightarrow N$ in $\mathscr{V}$ is called short exact if

$$
0 \rightarrow L_{[x]} \rightarrow M_{[x]} \rightarrow N_{[x]} \rightarrow 0
$$

is a short exact sequence of $S$-modules for any $x \in \mathscr{V}$.

Remark 5.3. This is not the original definition of exact structure Fiebig [2008a] gave, which was on the whole category $\mathscr{Z}$ - $\bmod ^{\mathrm{f}}$, but it is known to be equivalent to it if we only consider the category $\mathscr{V}$, that is, precisely the one we are dealing with (see [Fiebig 2008b, Lemma 2.12]).

Decomposition and subquotients of modules on $\mathscr{L} \mathbf{J}$. To show that $\mathscr{H}^{J}$ categorifies the parabolic Hecke algebra, we will use a description of the action of ${ }^{s} \theta$ on the subquotients $M_{[x]}$, for $x \in \mathscr{V}$ (Lemma 5.6). As a stepping-stone we prove an easy combinatorial consequence (Lemma 5.5) of the so-called lifting lemma:

Lemma 5.4 (lifting lemma [Humphreys 1990, Lemma 7.4]). Let $s \in \mathscr{Y}$ and $v, u \in \mathscr{W}$ be such that $v s<v$ and $u<v$.

(1) If $u s<u$, then $u s<v s$.

(2) If $u s>u$, then $u s \leq v$ and $u \leq v s$.

Thus in both cases, $u s \leq v$.

Lemma 5.5. Let $x \in \mathcal{W}^{J}$ and $t \in \mathscr{Y}$. If $t x \notin \mathscr{W}^{J}$, then $(t x)^{J}=x$.

Proof. If $t x \notin W^{J}$, then there exists a simple reflection $r \in J$ such that $t x r<t x$ and since $x \in \mathscr{W}^{J}, x r>x$. Using (the left version of) Lemma 5.4(1) with $s=t$, $v=x r$ and $u=t x$, we get $t x r<x$. Applying Lemma 5.4(1) with $s=r, v=x$ and $u=t x r$ it follows $t x>x$. Finally from Lemma 5.4(2) we obtain $t x r \leq x$, which together with $x<x r$, gives $t x r=x$. 
Lemma 5.6. Let $s \in \mathscr{S}$ and $x \in \mathscr{W}^{J}$; then

$$
\left({ }^{s} \theta M\right)_{[x]} \cong \begin{cases}M_{[x]}\langle-2\rangle \oplus M_{[s x]}\langle-2\rangle & \text { if } s x \in \mathscr{W}^{J}, s x>x, \\ M_{[x]} \oplus M_{[s x]} & \text { if } s x \in \mathscr{W}^{J}, s x<x, \\ M_{[x]}\langle-2\rangle \oplus M_{[x]} & \text { if } s x \notin \mathscr{W}^{J} .\end{cases}
$$

Proof. By Lemma 5.5, if $s x \notin \mathscr{W}^{J}$, then $(s x)^{J}=x$ and $M_{[x]} \in{ }^{s \mathscr{Z}}{ }^{J}$-mod; so by Lemma 4.1, we get $\mathscr{L}^{J} \otimes_{s o \mathscr{L} J} M_{[x]}=M_{[x]}\langle-2\rangle \oplus M_{[x]}$.

If $x \neq s x$, we have a short exact sequence of $S$-modules

$$
0 \rightarrow M_{[x]} \rightarrow M_{[x, s x]} \rightarrow M_{[s x]} \rightarrow 0 .
$$

By Lemma 4.1, the finitely generated free $S$-module $\mathscr{L}^{J}$ is flat over ${ }^{s} \mathscr{L}^{J}$, which is a finitely generated free $S^{\mathscr{W}_{J}}$-module. Hence ${ }^{s} \theta M_{[x, s x]}=\mathscr{L}^{J} \otimes_{s \mathscr{L} J} M_{x, s x}=$

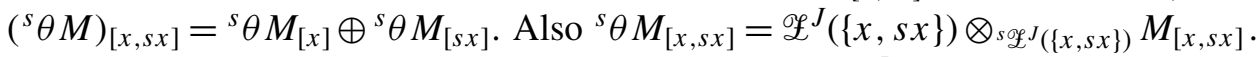
The two isomorphisms follow keeping in mind that $\mathscr{L}^{J}(\{x, s x\})_{[x]} \cong S\langle-2\rangle$ if $x<s x$, while $\mathscr{L}^{J}(\{x, s x\})_{[x]} \cong S$ if $x>s x$.

Using induction, we obtain the following corollary:

Corollary 5.7. Let $M \in \mathscr{H}^{J}$; then for any $x \in \mathscr{W}^{J}, M_{[x]}$ is a finitely generated torsion-free $S$-module and hence $M \in \mathscr{V}$.

In this way we get an exact structure on $\mathscr{H}^{J}$ as well and we are finally able to state the main result of this paper:

Theorem 5.8. The category $\mathscr{H}^{J}$ together with the shift in degree $\langle-1\rangle$ and (shifted) translation functors is a categorification of the parabolic Hecke module $\boldsymbol{M}^{J}$.

Remark 5.9. Theorem 5.8 could be strengthen to a proper categorification by presenting the result as a 2-representation of a 2-category. The 2-category to be considered is the one generated by the translation functors we defined on p. 426, and the 2-representation to look at is given by the action of these functors on the category $\mathscr{H}^{J}$ we constructed on p. 428. The question of describing indecomposable 1-morphisms in this category, which we are not going to address in this paper, seems to be very interesting.

Remark 5.10. It follows from [Elias and Williamson 2014] that the results of [Mazorchuk and Stroppel 2005] transfer to all Coxeter systems.

\section{Proof of the categorification theorem}

The proof of Theorem 5.8 consists of several steps:

(1) We show that the functor ${ }^{s} \theta \circ\langle 1\rangle$ is exact (Lemma 6.1).

(2) We define the character map $h^{J}:\left[\mathscr{H}^{J}\right] \rightarrow \boldsymbol{M}^{J}$ (p. 432). 
(3) We observe that the map $[\langle-1\rangle]:\left[\mathscr{H}^{J}\right] \rightarrow\left[\mathscr{H}^{J}\right]$ provides $\left[\mathscr{H}^{J}\right]$ with a structure of $\mathscr{L}$-module and that $h^{J}$ is a map of $\mathscr{L}$-modules (p. 432).

(4) Via explicit calculations, we prove that the functors ${ }^{s} \theta \circ\langle 1\rangle, s \in \mathscr{Y}$, satisfy (C3), that is, the maps they induce on $\left[\mathscr{H}^{J}\right]$ commute with $h^{J}$ (Proposition 6.2).

(5) We demonstrate that the character map is surjective by choosing a certain basis for $\boldsymbol{M}^{J}$ and showing that every element of this basis has a preimage in $\left[\mathscr{H}^{J}\right]$ under $h^{J}$ (Lemma 6.3).

(6) We prove that the character map is surjective (Lemma 6.6) using a description of indecomposable special modules in terms of Braden-MacPherson sheaves (Proposition 6.5).

This concludes the proof since $(\mathrm{C} 2)$, that is, $\langle-1\rangle \circ\left({ }^{s} \theta \circ\langle 1\rangle\right) \cong\left({ }^{s} \theta \circ\langle 1\rangle\right) \circ\langle-1\rangle$ for any $s \in \mathscr{Y}$, is trivially satisfied.

We start by proving the exactness of shifted translation functors.

Lemma 6.1. For any $s \in \mathscr{S}$ the functor ${ }^{s} \theta \circ\langle 1\rangle: \mathscr{H}^{J} \rightarrow \mathscr{H}^{J}$ is exact.

Proof. Let $L \rightarrow M \rightarrow N$ be an exact sequence; then for any $x \in \mathscr{V}$

$$
0 \rightarrow L_{[x]} \rightarrow M_{[x]} \rightarrow M_{[x]} \rightarrow 0
$$

is a short exact sequence of $S$-modules. In particular,

$$
0 \rightarrow L_{[s x]} \rightarrow M_{[s x]} \rightarrow N_{[s x]} \rightarrow 0
$$

is short exact as well. The claim follows immediately from Lemma 5.6 and the fact that finite direct sums and shifts preserve exactness.

Character maps. Let $A$ be a $\mathbb{Z}$-graded, free and finitely generated $S$-module; then $A \cong \bigoplus_{i=1}^{n} S\left\langle k_{i}\right\rangle$ for some $k_{i} \in \mathbb{Z}$. We can associate to $A$ its graded rank, that is, the following Laurent polynomial:

$$
\underline{\operatorname{rk}} A:=\sum_{i=1}^{n} v^{-k_{i}} \in \mathscr{L} .
$$

This is well defined because the $k_{i}$ are uniquely determined, up to reordering.

Let $M \in \mathscr{H}^{J}$. By Corollary 5.7, we may define a map $h^{J}:\left[\mathscr{H}^{J}\right] \rightarrow \boldsymbol{M}^{J}$ by

$$
h^{J}([M]):=\sum_{x \in \mathscr{W}^{J}} v^{\ell(x)} \underline{\mathrm{rk}} M_{[x]} H_{x}^{J, v^{-1}} \in M^{J} .
$$

The Grothendieck group $\left[\mathscr{H}^{J}\right]$ is equipped with a structure of $\mathscr{L}$-module via $v^{i}[M]=[M\langle-i\rangle]$. Observe that for any $M \in \mathscr{H}^{J}$

$$
h^{J}(v[M])=h^{J}([M\langle-1\rangle])=v h^{J}([M])
$$

and so $h^{J}$ is a map of $\mathscr{L}$-modules. 
Proposition 6.2. For each $M \in \mathscr{H}^{J}, s \in \mathscr{Y}$ we have $h^{J}\left(\left[{ }^{s} \theta M\langle 1\rangle\right]\right)=\underline{H}_{s} \cdot h^{J}([M])$; that is, the following diagram is commutative:

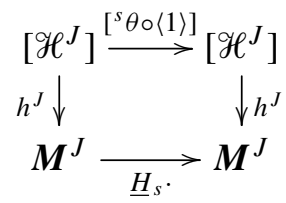

Proof. By Lemma 5.6, for any $x \in \mathcal{W}^{J}$ we have

$$
\underline{\mathrm{rk}}\left({ }^{s} \theta M\right)_{[x]}= \begin{cases}v^{2}\left(\underline{\mathrm{rk}} M_{[x]}+\underline{\mathrm{rk}} M_{[s x]}\right) & \text { if } s x \in \mathscr{W}^{J}, s x>x, \\ \underline{\mathrm{rk}} M_{[x]}+\underline{\mathrm{rk}} M_{[s x]} & \text { if } s x \in \mathscr{W}^{J}, s x<x, \\ \left(v^{2}+1\right) \underline{\mathrm{rk}} M_{[x]} & \text { if } s x \notin \mathscr{W}^{J} .\end{cases}
$$

Then

Finally

$$
\begin{aligned}
& h^{J}\left(\left[{ }^{s} \theta M\langle 1\rangle\right]\right)=\sum_{x \in \mathcal{W}^{J}} v^{\ell(x)-1} \underline{\mathrm{rk}}\left({ }^{s} \theta M\right)_{[x]} H_{x}^{J, v^{-1}} \\
& =\sum_{\substack{x \in W^{J}, s x \in \mathscr{W}^{J} \\
s x>x}} v^{\ell(x)+1}\left(\underline{\mathrm{rk}} M_{[x]}+\underline{\mathrm{rk}} M_{[s x]}\right) H_{x}^{J, v^{-1}} \\
& +\sum_{\substack{x \in \mathscr{W}^{J}, s x \in \mathscr{W}^{J} \\
s \dot{x}<x}} v^{\ell(x)-1}\left(\underline{\mathrm{rk}} M_{[x]}+\underline{\mathrm{rk}} M_{[s x]}\right) H_{x}^{J, v^{-1}} \\
& +\sum_{x \in \mathscr{W}^{J}, s x \notin \mathbb{W}^{J}}\left(v^{\ell(x)+1}+v^{\ell(x)-1}\right) \underline{\operatorname{rk}} M_{[x]} H_{x}^{J, v^{-1}} .
\end{aligned}
$$

$$
\begin{aligned}
\underline{H}_{s} \cdot h^{J}([M])= & \sum_{x \in \mathscr{W}^{J}} v^{\ell(x)}\left(\underline{\mathrm{rk}} M_{[x]}\right) \underline{H}_{s} \cdot H_{x}^{J, v^{-1}} \\
= & \sum_{x \in \mathscr{W}^{J}, s x \in \mathscr{W}^{J}} v^{\ell(x)}\left(\underline{\mathrm{rk}} M_{[x]}\right)\left(H_{s x}^{J, v^{-1}}+v H_{x}^{J, v^{-1}}\right) \\
& \left.+\sum_{x \in \mathscr{W}^{J}, s x \in \mathscr{W}^{J}} v^{\ell(x)} \underline{\mathrm{rk}} M_{[x]}\right)\left(H_{s x}^{J, v^{-1}}+v^{-1} H_{x}^{J, v^{-1}}\right) \\
& +\sum_{x \in \mathscr{W}^{J}, s x \notin^{J} \mathscr{W}^{J}} v^{\ell(x)} \underline{\mathrm{rk}} M_{[x]}\left(v+v^{-1}\right) H_{x}^{J, v^{-1}} \\
= & \sum_{x \in \mathscr{W}^{J}, s x \in \mathscr{W}^{J}}\left[\left(v^{\ell(x)} v \underline{\mathrm{rk}} M_{[x]}\right)+\left(v^{\ell(s x)} \underline{\mathrm{rk}} M_{[s x]}\right)\right] H_{x}^{J, v^{-1}} \\
& +\sum_{\substack{x \in \mathscr{W}^{J}, s x \in \mathscr{W}^{J} \\
s x<x}}\left[\left(v^{\ell(x)} v^{-1} \underline{\mathrm{rk}} M_{[x]}\right)+\left(v^{\ell(s x)} \underline{\mathrm{rk}} M_{[s x]}\right)\right] H_{x}^{J, v^{-1}} \\
& +\sum_{x \in \mathscr{W}^{J}, s x \notin^{J}}\left(v^{\ell(x)+1}+v^{\ell(x)-1}\right) \underline{\mathrm{rk}} M_{[x]} H_{x}^{J, v^{-1}}
\end{aligned}
$$




$$
=h^{J}\left(\left[{ }^{s} \theta M\langle 1\rangle\right]\right) \text {. }
$$

The character map is an isomorphism. In order to prove that $\left(\mathscr{H}^{J},\langle-1\rangle,\left\{{ }^{s} \theta \circ\right.\right.$ $\langle 1\rangle\})$ is a categorification of $\boldsymbol{M}^{J}$, the only step left is to show that $h^{J}$ is an isomorphism.

Lemma 6.3. The map $h^{J}:\left[\mathscr{H}^{J}\right] \rightarrow \boldsymbol{M}^{J}$ is surjective.

Proof. We start by defining a basis of $\boldsymbol{M}^{J}$. Let us set $\underline{\widetilde{H}}_{e}^{J, v^{-1}}=\underline{H}_{e}^{J, v^{-1}}$. For any $x \in \mathscr{W}^{J}$ with $\ell(x)=r>0$, let us fix a reduced $x=s_{i_{1}} \ldots s_{i_{r}}$, with $s_{i_{1}}, \ldots, s_{i_{r}} \in \mathscr{Y}$, and denote

$$
\underline{\widetilde{H}}_{x}^{J, v^{-1}}=\underline{H}_{s_{1}}^{J, v^{-1}} \cdots \underline{H}_{s_{r}}^{J, v^{-1}} .
$$

From Theorem 2.2, it follows that

$$
\underline{\widetilde{H}}_{x}^{J, v^{-1}}=H_{x}^{J, v^{-1}}+\sum_{\substack{y \in \mathcal{W}^{J} \\ y<x}} p_{y} H_{y}^{J, v^{-1}}, \quad \text { with } p_{z} \in \mathbb{Z}\left[v, v^{-1}\right] .
$$

Since $\left\{H_{x}^{J, v^{-1}}\right\}_{x \in W^{J}}$ is a basis of $\boldsymbol{M}^{J}$ as a $\mathbb{Z}\left[v, v^{-1}\right]$-module, $\left\{\underline{\tilde{H}}_{x}^{J, v^{-1}}\right\}_{x \in \mathscr{W}^{J}}$ is also a basis for $\boldsymbol{M}^{J}$ and it is enough to show that, for any $x \in \mathcal{W}^{J}$, there exists an object $H \in \mathscr{H}^{J}$ such that $h^{J}([H])=\underline{\widetilde{H}}_{x}^{J, v^{-1}}$.

By definition, $h^{J}\left(B_{e}^{J}\right)=M_{e}=\underline{H}_{e}^{J, v^{-1}}$. By applying Proposition 6.2, we obtain

$$
\begin{aligned}
h^{J}\left({ }^{s_{1}} \theta \circ \cdots \circ \circ^{s_{i_{r}}} \theta B_{e}^{J}\langle n\rangle\right) & =\left(\underline{H}_{s_{1}}^{J, v^{-1}} \cdots \underline{H}_{s_{r}}^{J, v^{-1}}\right) M_{e} \\
& =\underline{H}_{s_{1}}^{J, v^{-1}} \cdots \underline{H}_{s_{r}}^{J, v^{-1}}=\underline{\widetilde{H}}_{x}^{J, v^{-1}} .
\end{aligned}
$$

This concludes the proof of the lemma.

Proposition 6.5 will allow us to see any element in $\mathscr{H}^{J}$ as the space of global sections of some BMP sheaf on $\varphi^{J}$. From now on, we will denote by $B^{J}(w)$ the space of global sections of the indecomposable BMP sheaf $\mathscr{B}^{J}(w) \in \mathbf{S h}\left(\varphi^{J}\right)$. Let us recall a fundamental characterisation of $B^{J}(w)$.

Theorem 6.4 [Fiebig 2008b, Theorem 5.2]. For any $w \in \mathscr{G}^{J}$, the module $B^{J}(w) \in \mathscr{V}$ is indecomposable and projective. Moreover every indecomposable projective object in $\mathscr{V}$ is isomorphic to $B^{J}(w)\langle k\rangle$ for a unique $w \in \mathscr{G}^{J}$ and a unique $k \in \mathbb{Z}$.

Proposition 6.5. A module $M \in \mathscr{L}^{J}$-mod mo $^{f}$ an indecomposable special module if and only if there exist a $B M P$ sheaf $\mathscr{B} \in \boldsymbol{S h}\left(\varphi^{J}\right)$ and $k \in \mathbb{Z}$ such that $M \cong \Gamma(\mathscr{B}\langle k\rangle)$ as $\mathscr{L}^{J}$-modules.

Proof. By induction, from the exactness of ${ }^{s} \theta^{J}$, it follows that the objects of $\mathscr{H}^{J}$ are all projective, and then by Theorem 6.4, any $M \in \mathscr{H}^{J}$ may be identified (up to a shift) with the space of global sections of a BMP sheaf on $\varphi^{J}$.

We now want to show that for any $x \in \mathscr{W}_{J}, B^{J}(x) \in \mathscr{H}^{J}$. We prove the claim by induction on $\# \operatorname{supp}(M)$, where $\operatorname{supp}(M)=\left\{x \in \mathcal{W}^{J} \mid M^{x} \neq 0\right\}$. Clearly $B_{e} \cong B^{J}(e)$. 
The statement follows straightforwardly, once we prove that if $s x>x$, then ${ }^{s} \theta^{J}\left(B^{J}(x)\right)=B^{J}(s x) \oplus B$.

First we show that $\operatorname{supp}\left({ }^{s} \theta^{J}\left(B^{J}(x)\right)\right) \subseteq\{\leq s x\}$, that is, $\left({ }^{s} \theta^{J} B^{J}(x)\right)^{y}=0$ for all $y \notin\{\leq s x\} \cap \mathcal{W}^{J}$. From Lemma 5.6, it follows easily that $\left({ }^{s} \theta^{J}\left(B^{J}(x)\right)\right)_{[y]}=0$ for all $y \notin\{\leq s x\} \cap W^{J}$.

Let us observe that as ${ }^{s} \theta^{J} B^{J}(x) \in \mathscr{H}^{J}$, from what we have proved above, there exist $w_{1}, \ldots, w_{r} \in \mathcal{W}^{J}$ and $k_{1}, \ldots, k_{r}$ such that ${ }^{s} \theta^{J}\left(B^{J}(x)\right)=\bigoplus_{i=1}^{r} B^{J}\left(w_{i}\right)\left\langle k_{i}\right\rangle$, and for any $y \in W^{J}$,

$$
\left(\bigoplus_{i=1}^{r} B^{J}\left(w_{i}\right)\left\langle k_{i}\right\rangle\right)_{[y]}=\bigoplus_{i=1}^{r} B^{J}\left(w_{i}\right)_{[y]}\left\langle k_{i}\right\rangle .
$$

So, in particular, for all $y \notin\{\leq s x\} \cap W^{J}$,

$$
\begin{aligned}
0 & =B^{J}\left(w_{i}\right)_{[y]} \\
& =\operatorname{ker}\left(\rho_{\delta y}: \mathscr{B}^{J}\left(w_{i}\right)^{y} \rightarrow \mathscr{B}^{J}\left(w_{i}\right)^{\delta y}\right) .
\end{aligned}
$$

This implies $\mathscr{B}^{J}\left(w_{i}\right)^{y}=B^{J}\left(w_{i}\right)^{y}=0$ for all $i=1, \ldots r$, and so

$$
{ }^{s} \theta^{J}\left(B^{J}(x)\right)=\bigoplus_{i=1}^{r} B^{J}\left(w_{i}\right)\left\langle k_{i}\right\rangle,
$$

where $w_{i} \in\{\leq s x\}$ for all $i=1, \ldots, r$.

It is left to show that there exists at least one $i \in\{1, \ldots, r\}$ such that $w_{i}=s x$. By applying once again Lemma 5.6, we obtain $\left({ }^{s} \theta^{J}\left(B^{J}(x)\right)\right)^{s x}=\left({ }^{s} \theta^{J}\left(B^{J}(x)\right)\right)_{[s x]} \cong S$ and hence the statement.

Lemma 6.6. The map $h^{J}:\left[\mathscr{H}^{J}\right] \rightarrow M^{J}$ is injective.

Proof. By Theorem 6.4 and Proposition 6.5 we know that $\left\{\left[B^{J}(w)\right]\right\}_{w \in^{\circ} W^{J}}$ is a $\mathbb{Z}\left[v, v^{-1}\right]$-basis of $\left[\mathscr{H}^{J}\right]$ and so every element $Y \in\left[\mathscr{H}^{J}\right]$ can be written as $Y=\sum a_{w}\left[B^{J}(w)\right]$, with $a_{x} \in \mathbb{Z}\left[v, v^{-1}\right]$. Let us suppose $Y \in \operatorname{ker}\left(h^{J}\right)$. Then

$$
\begin{aligned}
0=h^{J}(Y) & =\sum_{w \in \mathscr{W}^{J}} a_{w} \sum_{x \in \mathcal{W}^{J}} v^{\ell(x)} \underline{\mathrm{rk}} B^{J}(w)_{[x]} H_{x}^{J, v^{-1}} \\
& =\sum_{x \in \mathscr{W}^{J}}\left(\sum_{w \in \mathcal{W}^{J}} v^{\ell(x)} a_{w} \underline{\mathrm{rk}} B^{J}(w)_{[x]}\right) H_{x}^{J, v^{-1}} .
\end{aligned}
$$

Since the elements $H_{x}^{J, v^{-1}}$ are linearly independent, it follows that

$$
\sum_{w \in \mathscr{W}^{J}} v^{\ell(x)} a_{w} \underline{\mathrm{rk}} B^{J}(w)_{[x]}=0 \quad \text { for any } x \in \mathscr{W}^{J} .
$$

If it were the case that $Y \neq 0$, then we would find a maximal element $\bar{w}$ such that 
$a_{\bar{w}} \neq 0$. By $\left(\right.$ BMP1), we obtain $B^{J}(w)_{[\bar{w}]}=0$ for all $w<\bar{w}$ and $B^{J}(\bar{w})_{[\bar{w}]} \cong S$. Then

$$
0=\sum_{w \in \mathcal{W}^{J}} v^{\ell(x)} a_{w} \underline{\mathrm{rk}} B^{J}(w)_{[\bar{w}]}=v^{\ell(x)} a_{\bar{w}} \underline{\mathrm{rk}} B^{J}(\bar{w})_{[\bar{w}]}=v^{\ell(x)} a_{\bar{w}} \underline{\mathrm{rk}} S=v^{\ell(x)} a_{\bar{w}} .
$$

The chain of equalities above gives us a contradiction since we assumed $a_{\bar{w}} \neq 0$.

This concludes the proof of Theorem 5.8.

\section{The functor $I$}

In this section we define an exact functor $I: \mathscr{H}^{J} \rightarrow \mathscr{H}^{\varnothing}$ such that the following diagram commutes:

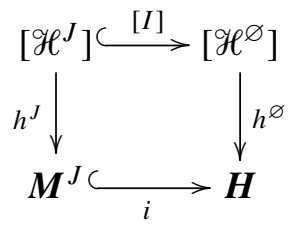

where $i: \boldsymbol{M}^{J} \hookrightarrow \boldsymbol{H}$ is the map of $\mathscr{L}$-modules given by

$$
H_{x}^{J, v^{-1}} \mapsto \sum_{z \in \mathscr{W}_{J}} v^{\ell\left(w_{J}\right)-\ell(z)} H_{x z},
$$

with $w_{J}$ the longest element of $\mathcal{W}_{J}$.

The map $i$ is interesting since it gives us a way to see the parabolic Hecke module $\boldsymbol{M}^{J}$ as submodule of $\boldsymbol{H}$, and hence its categorification tells us that we can think about the $\mathscr{H}^{J}$ as a subcategory of $\mathscr{H}^{\varnothing}$.

We construct the functor $I$ by using a localisation-globalisation procedure. More precisely, we first map the elements of $\mathscr{H}^{J}$ to certain sheaves on $\mathscr{G}^{J}$, then apply a pullback functor mapping them to sheaves on $\mathscr{G}$, and finally we take global sections of the latter. A priori it is not clear that we obtain an object in $\mathscr{H}^{\varnothing}$. This fact is shown in Lemma 7.3. We then demonstrate the exactness of $I$ (Proposition 7.5) and the commutativity of diagram 7 (Proposition 7.7) by a study of the subquotients involved in the definition of the character map. The realisation of special modules in terms of Braden-MacPherson sheaves given in the previous section (Proposition 6.5) plays a crucial role in the proof of any of the above results.

Construction of the functor $I$. The definition of $I$ involves Fiebig's localisation functor $\mathscr{L}$ [2008a, §3.3], which allows us to see objects of $\mathscr{L}^{J}$-mod as sheaves on the parabolic Bruhat moment graph $\mathscr{G}^{J}$.

Let us assume $\mathscr{G}$ to be such that for any $M \in \mathscr{L}$-mod ${ }^{\mathrm{f}}$ there is a canonical decomposition like the one in (7). Let $\mathscr{L}$ be the corresponding structure algebra 
and $M \in \mathscr{L}$-mod ${ }^{\mathrm{f}}$. For any vertex $x \in \mathscr{V}$, we set

$$
\mathscr{L}(M)^{x}=M^{x} .
$$

For any edge $E: x-y$, let us consider $\mathscr{L}(E)=\left\{\left(z_{x}, z_{x}\right) \in S \oplus S \mid z_{x}-z_{y} \in l(E) S\right\}$ and $M(E):=\mathscr{L}(E) \cdot M^{x, y}$. For $m=\left(m_{x}, m_{y}\right) \in M(E)$, let us set $\pi_{x}((m))=m_{x}$, $\pi_{y}((m))=m_{y}$. Then we get $\mathscr{L}(M)^{E}$ as the pushout in the following diagram of $S$-modules:

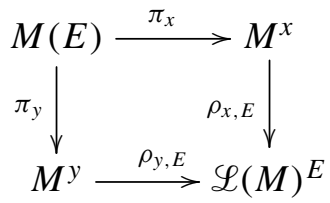

This provides us also with the restriction maps $\rho_{x, E}$ and $\rho_{y, E}$.

It is not hard to verify (see [Fiebig 2008a, §3.3]) that this is a well defined functor

$$
\mathscr{L}: \mathscr{L}-\bmod ^{\mathrm{f}} \rightarrow \mathbf{S h}(\mathscr{G}) .
$$

Moreover the localisation functor $\mathscr{L}$ turns out to be left-adjoint to $\Gamma$ (see [Fiebig 2008a, Theorem 3.5]). Let

- $\mathscr{L}$-mod ${ }^{\text {loc }}$ be the full subcategory of $\mathscr{L}$-mod ${ }^{\mathrm{f}}$ whose objects are the elements $M$ such that there is an isomorphism $\Gamma \circ \mathscr{L}(M) \cong M$, and

- $\mathbf{S h}(\mathscr{G})^{\text {glob }}$ be the full subcategory of $\mathbf{S h}(\mathscr{G})$ whose objects are the elements $\mathscr{F}$ such that there is an isomorphism $\mathscr{L} \circ \Gamma(\mathscr{F}) \cong \mathscr{F}$.

Remark 7.1. In general, for a given a sheaf $\mathscr{F}$, one has $(\mathscr{L} \circ \Gamma(\mathscr{F}))^{x}=\Gamma\left(\mathscr{F}^{x}\right)^{x} \subseteq \mathscr{F}^{x}$. If we consider a BMP sheaf $\mathscr{R}$, then by property (BMP3), $\Gamma(\mathscr{B})^{x}=\mathscr{B}^{x}$ for any vertex $x \in \mathscr{V}$ and $\mathscr{L}(\Gamma(\mathscr{B}))^{E} \cong \mathscr{B}^{E}$ for any edge $E \in \mathscr{E}$. Therefore $\mathscr{L} \circ \Gamma(\mathscr{B}) \cong \mathscr{B}$ and $\mathscr{B} \in \mathbf{S h}(\varphi)^{\text {glob }}$.

Thus the functors $\mathscr{L}$ and $\Gamma$ induce two inverse equivalences:

$$
\mathscr{L}-\bmod ^{\mathrm{loc}} \longleftrightarrow \mathbf{S h}(\mathscr{\varphi})^{\mathrm{glob}} .
$$

Let us focus again on the Bruhat case and consider the functor

$$
p^{J, *}: \mathbf{S h}\left(\varphi^{J}\right) \rightarrow \mathbf{S h}\left(\varphi^{\prime}\right)
$$

defined as follows:

- $\left(p^{J, * \mathscr{F}^{x}}\right)^{x}:=\mathscr{F}^{x^{J}}$ for all $x \in \mathscr{W}$.

- for all $E: x-y \in \mathscr{E}$,

$$
\left(p^{J, * \mathscr{F}_{F}}\right)^{E}= \begin{cases}\mathscr{F}_{\mathscr{F}} f_{\mathcal{V}}(x) / l(E) \mathscr{F}_{f} f_{\mathcal{V}}(x) & \text { if } x^{J}=y^{J}, \\ \mathscr{F}_{\mathscr{F}} f_{\mathscr{E}}(E) & \text { otherwise } .\end{cases}
$$


- for all $x \in \mathscr{W}$ and $E \in \mathscr{E}$ such that $E: x-y$,

$$
\left(p^{J, *} \rho\right)_{x, E}= \begin{cases}\text { canonical quotient map } & \text { if } x^{J}=y^{J}, \\ \rho_{f_{\mathcal{V}}(x), f_{\mathscr{E}}(E)} & \text { otherwise } .\end{cases}
$$

Finally we set $I:=\left\langle-\ell\left(w_{J}\right)\right\rangle \circ \Gamma \circ p^{J, *} \circ \mathscr{L}$.

To prove that the functor $I$ maps $\mathscr{H}^{J}$ to $\mathscr{H}$, we need to recall the moment graph analogue of a theorem by Deodhar relating parabolic Kazhdan-Lusztig polynomials and regular ones. The following is a reformulation of Theorem 6.1 of [Lanini 2012]:

Theorem 7.2. Let $J \subseteq \mathscr{9}$ be such that $W_{J}$ is finite, with longest element $w_{J}$. Let $w \in \mathscr{W}^{J}$; then $p^{J, *}\left(\mathscr{B}^{J}(w)\right) \cong \mathscr{B}^{\varnothing}\left(w w_{J}\right)$ as sheaves on $\mathscr{G}=\mathscr{G}(\mathcal{W}, \varnothing)$.

Lemma 7.3. The functor I maps $\mathscr{H}^{J}$ to $\mathscr{H}$.

Proof. Let $M \in \mathscr{H}^{J}$; then, by Proposition 6.5, there exist $w_{1}, \ldots w_{r} \in \mathscr{W}^{J}$ and $m_{1}, \ldots m_{r} \in \mathbb{Z}$ such that $M=\bigoplus_{i=1}^{r} B^{J}\left(w_{i}\right)\left\langle m_{i}\right\rangle$. Then

$$
I(M)=I\left(\bigoplus_{i=1}^{r} B^{J}\left(w_{i}\right)\left\langle m_{i}\right\rangle\right)=\bigoplus_{i=1}^{r} \Gamma \circ p^{J, *} \circ \mathscr{L}\left(B^{J}\left(w_{i}\right)\right)\left\langle m_{i}-\ell\left(w_{J}\right)\right\rangle .
$$

By Remark 7.1, $\mathscr{L}\left(B^{J}\left(w_{i}\right)\right) \cong \mathscr{B}^{J}\left(w_{i}\right)$ for any $i$ and, by Theorem 7.2, we conclude that

$$
I(M) \cong \bigoplus_{i=1}^{r} B^{\varnothing}\left(w_{i} w_{J}\right)\left\langle m_{i}-\ell\left(w_{J}\right)\right\rangle
$$

\section{Exactness of $I$.}

Lemma 7.4. Let $w \in \mathcal{W}^{J}$. Then for all $x \in \mathcal{W}$,

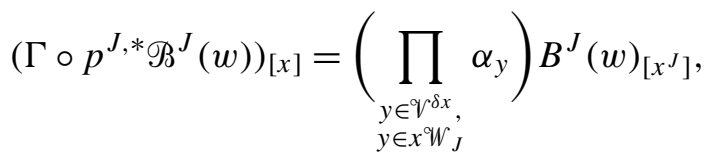

where $\alpha_{y}$ denotes the label of $x \rightarrow y$.

Proof. For $z \in \mathcal{W}^{J}$ and $E$ an edge of $\mathscr{G}^{J}=\mathscr{G}(\mathcal{W}, J)$, let us denote by $\rho_{z, E}$ the corresponding restriction map. Then we have

$$
\begin{aligned}
\left(\Gamma \circ p^{J, * \mathscr{B}^{J}}(w)\right)_{[x]} & =\bigcap_{y \in \mathcal{V}^{\delta x}} \operatorname{ker}\left(\left(p^{*, J} \rho\right)_{x, x \rightarrow y}\right) \\
& =\left(\bigcap_{\substack{y \in \mathcal{V}^{\delta x} \\
y \notin \mathcal{W}_{J}}} \operatorname{ker}\left(\rho_{x^{J}, x^{J} \rightarrow y^{J}}\right)\right) \cap\left(\bigcap_{\substack{y \in \mathcal{V}^{\delta x}, y \in x^{\prime} W_{J}}} \operatorname{ker} \pi_{x, x \rightarrow y}\right),
\end{aligned}
$$

where $\pi_{x, x \rightarrow y}: \mathscr{B}^{J}(w)^{x^{J}} \rightarrow \mathscr{B}^{J}(w)^{x^{J}} / \alpha_{y} \mathscr{B}^{J}(w)^{x^{J}}$ is the canonical quotient map and $\alpha_{y}$ is a generator of $l(x \rightarrow y)$. 
Let us observe that by definition,

$$
\bigcap_{\substack{y \in \mathcal{V}^{\delta x} \\ y \notin x \mathcal{W}_{J}}} \operatorname{ker}\left(\rho_{x^{J}, x^{J} \rightarrow y^{J}}\right)=B^{J}(w)_{\left[x^{J}\right]} .
$$

Moreover since there is at most one edge adjacent to $x$ labelled by a multiple of $\alpha_{y}$, the labels of such edges are pairwise linearly independent and we get

$$
\bigcap_{\substack{y \in \mathcal{V}^{\delta x}, y \in x^{W_{J}}}} \operatorname{ker} \pi_{x, x \rightarrow y}=\prod_{\substack{y \in \mathcal{V}^{\delta x}, y \in \mathcal{W}_{J}}} \alpha_{y} \cdot \mathscr{B}^{J}(w)^{x^{J}} .
$$

It follows that

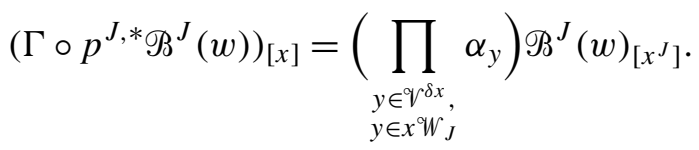

This concludes the proof of the lemma.

Proposition 7.5. The functor I is exact with respect to the exact structure in Section 5.

Proof. Let us take $M, N \in \mathscr{H}^{J}$, with $M=\bigoplus_{k} B^{J}\left(w_{k}\right)\left\langle m_{k}\right\rangle$ and $N=\bigoplus_{l} B^{J}\left(w_{l}\right)\left\langle n_{l}\right\rangle$.

Let us consider the map $f: L \rightarrow M$ and the induced maps $f_{\left[x^{J}\right]}: M_{\left[x^{J}\right]} \rightarrow N_{\left[x^{J}\right]}$ for any $x^{J} \in \mathcal{W}^{J}$. Thanks to Lemma 7.4, it is easy to describe $I(f)_{[x]}$. Namely if

$$
\prod_{\substack{y \in \mathcal{V}^{\delta x} \\ y \in x^{W} W_{J}}} \alpha_{y}=\alpha_{i_{1}} \cdots \alpha_{i_{r}},
$$

we obtain

$$
\begin{aligned}
& I(f): \quad I(M)_{[x]} \quad \longrightarrow \quad I(N)_{[x]}, \\
& \left(\alpha_{i_{1}} \cdots \alpha_{i_{r}}\right) m \longmapsto\left(\alpha_{i_{1}} \cdots \alpha_{i_{r}}\right) f_{[x]}(m) \text {. }
\end{aligned}
$$

It is clear that if $0 \rightarrow L_{[x]} \rightarrow M_{[x]} \rightarrow N_{[x]} \rightarrow 0$ is a short exact sequence of $S$-modules, then $0 \rightarrow(I L)_{[x]} \rightarrow(I M)_{[x]} \rightarrow(I N)_{[x]} \rightarrow 0$ is also exact.

Commutativity of the diagram. The last step missing is the commutativity of Diagram 7. Before proving it, we need the following preliminary lemma:

Lemma 7.6. Let $w \in W^{J}$ and let $w_{J}$ be the longest element of $\mathscr{W}_{J}$. There is an isomorphism $B^{\varnothing}\left(w w_{J}\right)_{[x]} \cong B^{J}(w)_{\left[x^{J}\right]}\left\langle 2 \ell(x)-2 \ell\left(x^{J}\right)-2 \ell\left(w_{J}\right)\right\rangle$ of graded $S$-modules.

Proof. By Theorem 7.2, $\mathscr{B}^{\varnothing}\left(w w_{J}\right) \cong p^{J, * \mathscr{B}^{J}}\left(w_{J}\right)$ as sheaves on $\mathscr{G}=\mathscr{G}(\mathscr{W}, \varnothing)$. It follows that for any $x \in \mathscr{W}, B^{\varnothing}\left(w w_{J}\right)_{[x]} \cong\left(\Gamma \circ p^{J, * \mathscr{B}^{J}}\left(w_{J}\right)\right)_{[x]}$ as graded 
$S$-modules and then, by Lemma 7.4, we obtain

$$
\begin{aligned}
B^{\varnothing}\left(w w_{J}\right)_{[x]} & \cong\left(\prod_{\substack{y \in \mathscr{V}^{\delta x}, y \in \mathcal{W}_{J}}} \alpha_{y}\right) B^{J}(w)_{\left[x^{J}\right]} \\
& \cong B^{J}(w)_{\left[x^{J}\right]}\left\langle 2 \cdot \#\left\{y \in \mathscr{V}^{\delta x}, y \in x \mathcal{W}_{J}\right\}\right\rangle .
\end{aligned}
$$

Let $x^{\prime}=\left(x^{J}\right)^{-1} x \in W_{J}$. Now if $\mathscr{T}_{J}$ is the set of reflections of $\mathscr{W}_{J}$, $\#\left\{y \in \mathscr{V}^{\delta x}, y \in x \mathscr{W}_{J}\right\}=\#\left\{z \in \mathscr{W}_{J} \mid\right.$ there exists $t \in \mathscr{T}_{J}$ s.t. $z=x^{\prime} t$ and $\left.x^{\prime}<z\right\}$

$$
=\ell\left(w_{J}\right)-\ell\left(x^{\prime}\right)=\ell\left(w_{J}\right)-\ell(x)+\ell\left(x^{J}\right) .
$$

Finally we are able to prove the following proposition, which enables us to embed $\mathscr{H}^{J}$ in $\mathscr{H}$.

Proposition 7.7. The following diagram is commutative:

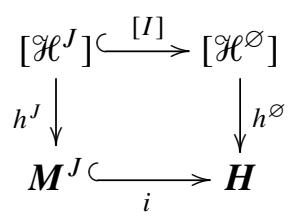

Proof. As $I\left(\bigoplus_{i \in I} B^{J}\left(w_{i}\right)\right)=\bigoplus I\left(B^{J}\left(w_{i}\right)\right)$, it is enough to prove the statement for the module $B^{J}(w)$. In this case, we have:

$$
\begin{aligned}
I\left(B^{J}(w)\right) & =\left\langle-\ell\left(w_{J}\right)\right\rangle \circ \Gamma \circ p^{J, *} \circ \mathscr{L}\left(B^{J}(w)\right) \\
& =\left\langle-\ell\left(w_{J}\right)\right\rangle \circ \Gamma \circ p^{J, *}\left(\mathscr{B}^{J}(w)\right) \\
& \cong\left\langle-\ell\left(w_{J}\right)\right\rangle \circ \Gamma\left(\mathscr{B}^{\varnothing}\left(w w_{J}\right)\right) \\
& =B\left(w w_{J}\right)\left\langle-\ell\left(w_{J}\right)\right\rangle .
\end{aligned}
$$

Thus if $B^{J}(w)_{\left[x^{J}\right]}=\bigoplus_{i \in I_{x} J} S\left\langle k_{i}\right\rangle$, we get

$$
\begin{aligned}
h^{\varnothing} \circ[I]\left(\left[B^{J}(w)\right]\right) & =h^{\varnothing}\left(B^{\varnothing}\left(w w_{J}\right)\left\langle\ell\left(w_{J}\right)\right\rangle\right) \\
& =\sum_{x \in \mathscr{W}} v^{-\ell\left(w_{J}\right)+\ell(x)} \underline{\mathrm{rk}} B^{\varnothing}\left(w w_{J}\right)_{[x]} H_{x} \\
(\text { by Lemma 7.6) } & =\sum_{x \in \mathscr{W}} v^{\ell\left(w_{J}\right)+\ell(x)} \underline{\mathrm{rk}}\left(B^{J}(w)_{\left[x^{J}\right]}\left\langle 2 \ell\left(x_{J}\right)-2 \ell\left(w_{J}\right)\right\rangle\right) H_{x} \\
& =\sum_{x \in \mathscr{W}} v^{-\ell\left(w_{J}\right)+\ell(x)}\left(\sum_{i \in I_{x}} v^{-2 \ell\left(x_{J}\right)+2 \ell\left(w_{J}\right)-k_{i}}\right) H_{x} \\
& =\sum_{x \in \mathscr{W}} v^{\ell\left(w_{J}\right)+\ell(x)}\left(\sum_{i \in I_{x^{J}}} v^{-2 \ell\left(x_{J}\right)-k_{i}}\right) H_{x},
\end{aligned}
$$


where $H_{x}=H_{x}^{\varnothing, v^{-1}}$. On the other hand, we have

$$
\begin{aligned}
i \circ h^{J}\left(\left[B^{J}(w)\right]\right) & =i\left(\sum_{x^{J} \in \mathscr{W}^{J}} v^{\ell\left(x^{J}\right)} \underline{\mathrm{rk}} B^{J}(w)_{\left[x^{J}\right]} H_{x^{J}}^{J, v^{-1}}\right) \\
& =\sum_{x^{J} \in \mathscr{W}^{J}}\left[v^{\ell\left(x^{J}\right)}\left(\sum_{i \in I_{x^{J}}} v^{-k_{i}}\right) i\left(H_{x^{J}}^{J, v^{-1}}\right)\right] \\
& =\sum_{x^{J} \in \mathscr{W}^{J}}\left[v^{\ell\left(x^{J}\right)}\left(\sum_{i \in I_{x^{J}}} v^{-k_{i}}\right)\left(\sum_{x_{J} \in \mathscr{W}_{J}} v^{\ell\left(w_{J}\right)-l\left(x_{J}\right)} H_{x^{J} x_{J}}\right)\right] \\
& =\sum_{x^{J} \in \mathscr{W}^{J}} \sum_{x_{J} \in \mathscr{W}_{J}}\left(\sum_{i \in I_{x} J} v^{\ell\left(x^{J}\right)-k_{i}+\ell\left(w_{J}\right)-\ell\left(x_{J}\right)}\right) H_{x^{J} x_{J}} \\
& =\sum_{x \in \mathscr{W}^{\prime}} v^{\ell\left(w_{J}\right)+\ell(x)}\left(\sum_{i \in I_{x^{J}}} v^{-2 \ell\left(x_{J}\right)-k_{i}}\right) H_{x} .
\end{aligned}
$$

\section{Connection with the equivariant category 0}

In this section, we briefly discuss the connection of our results with noncritical blocks in an equivariant version of category $\mathbb{O}$.

Let $\mathfrak{g}$ be a complex symmetrisable Kac-Moody algebra and $\mathfrak{b} \supseteq \mathfrak{h}$ its Borel and Cartan subalgebras. The Weyl group $\mathscr{W}$ of $\mathfrak{g}$ naturally acts on $\mathfrak{h}^{\star}$, and we can consider equivalence classes $\Lambda \in \mathfrak{h}^{\star} / \sim$. An element $\lambda \in \mathfrak{h}^{\star}$ is noncritical if $2(\lambda+\rho, \beta) \notin \mathbb{Z}(\beta, \beta)$ for any imaginary root $\beta$, and an orbit $\Lambda$ is noncritical if any $\lambda \in \Lambda$ is noncritical.

Fix a noncritical orbit $\Lambda$ and a weight $\lambda_{0} \in \Lambda$. As in Definition 3.3, we can look at the $\mathscr{W}$-orbit of $\lambda_{0}$, which gives us a Bruhat moment graph on $\mathfrak{h}^{\star}$. We want to discuss the representation theoretic content of $\mathscr{H}^{J}$, where $J$ is in this case given by the set of simple reflections generating $\operatorname{Stab}_{W} \lambda_{0}$. Denote by $\mathscr{G}(\Lambda)$ such a graph.

Let $S=S(\mathfrak{h})$ be the symmetric algebra of $\mathfrak{h}, R=S_{(\mathfrak{h})}$ be its localisation at $0 \in \mathfrak{h}^{\star}$ and $\tau: S \rightarrow R$ be the canonical map. For any $\mu \in \mathfrak{h}^{\star}$ and any $(\mathfrak{g}-R)$-bimodule $M$, we define its $\mu$-weight space as

$$
M_{\mu}=\{m \in M \mid H . m=(\lambda(H)+\tau(H)) m \text { for any } H \in \mathfrak{h}\} .
$$

If $\mathfrak{g}$-mod- $R$ denotes the category of $(\mathfrak{g}-R)$-bimodules, then the equivariant version of category 0 we want to study is

$$
\mathrm{O}_{R}=\left\{\begin{array}{l|l}
M \in \mathfrak{g} \text {-mod- } R & \begin{array}{l}
M \text { is locally finite as a }(\mathfrak{b}-R) \text {-bimodule, } \\
M=\bigoplus_{\mu \in \mathfrak{h}^{\star}} M_{\mu}
\end{array}
\end{array}\right\} .
$$

For any $\mu \in \mathfrak{h}^{\star}$ let us consider the (h- $R$ )-bimodule $R_{\mu}$ free of rank one over $R$ on which $\mathfrak{h}$ acts via the character $\mu+\tau$. The projection $\mathfrak{b} \rightarrow \mathfrak{h}$ allows us to consider $R_{\mu}$ as a $(\mathfrak{b}-R)$-bimodule and we can now induce to obtain the equivariant Verma module 
of weight $\mu: M_{R}(\mu)=U(\mathfrak{g}) \otimes_{U(\mathfrak{b})} R_{\mu}$, where $U(\mathfrak{g})$ and $U(\mathfrak{b})$ are the enveloping algebras of $\mathfrak{g}$ and $\mathfrak{b}$, respectively.

Let $M_{R}$ be the full subcategory of $\mathscr{O}_{R}$ whose objects admit a finite filtration with subquotients isomorphic to equivariant Verma modules. Since $\mathbb{O}_{R}$ is abelian and $\mu_{R}$ is closed under extensions in $O_{R}$, the category $\mu_{R}$ inherits an exact structure.

For an equivalence class $\Lambda \in \mathfrak{h}^{\star} / \sim$, let $\mathcal{O}_{R, \Lambda}$, respectively, $\mathcal{M}_{R, \Lambda}$, be the full subcategory of $\mathcal{O}_{R}$, respectively, $M_{R}$, consisting of all objects $M$ such that the highest weight of every simple subquotient of $M$ lies in $\Lambda$. Then there are block decompositions, according to the following two results.

Proposition 8.1 [Fiebig 2003, Proposition 2.8; Fiebig 2008a, Theorem 6.1]. The functors

$$
\prod_{\Lambda \in \mathfrak{h}^{\star} / \sim} \mathcal{O}_{R, \Lambda} \rightarrow \mathcal{O}_{R}, \quad\left\{M_{\Lambda}\right\} \mapsto \bigoplus_{\Lambda \in \mathfrak{h}^{\star} / \sim} M_{\Lambda}
$$

and

$$
\prod_{\Lambda \in \mathfrak{h}^{\star} / \sim} \mu_{R, \Lambda} \rightarrow M_{R}, \quad\left\{M_{\Lambda}\right\} \mapsto \bigoplus_{\Lambda \in \mathfrak{h}^{\star} / \sim} M_{\Lambda}
$$

are equivalences of categories.

Now it is important to notice that we could have substituted $S$ by the local algebra $R$ in the constructions and definitions we have considered, and all the results of this paper would have still worked. Let us denote by $\mathscr{L}_{R}$ the $R$-version of the structure algebra of $\mathscr{G}(\Lambda)$ and by $\mathscr{V}_{R, \Lambda}$ the category of $\mathscr{L}_{R}$-modules admitting a Verma flag. The main result of [Fiebig 2008a] is the following one:

Theorem 8.2 [Fiebig 2008a, Theorem 7.1]. There is an equivalence of exact categories

$$
\mathbb{V}: \mu_{R, \Lambda} \rightarrow \mathscr{V}_{R, \Lambda}
$$

Projective objects. For $v \in \Lambda$, let $\Lambda^{\leq v}:=\{\lambda \in \Lambda \mid \lambda \leq v\}$. We want to consider a truncated version of $\mathcal{M}_{R, \Lambda}$ :

$$
M_{R, \Lambda \leq \nu}=\left\{M \in M_{R, \Lambda} \mid\left(M: M_{R}(\mu)\right) \neq 0 \text { only if } \mu \in \Lambda^{\leq \nu}\right\} .
$$

As a reference for the truncated category $\mathbb{O}$, we address the reader to [Rocha-Caridi and Wallach 1982], where it was introduced.

Denote by $\mathscr{V}_{R, \Lambda \leq \nu}$ the category of sheaves on the moment graph $\mathscr{G}(\Lambda)^{\leq \nu}$, obtained by restricting the set of vertices of $\mathscr{G}(\Lambda)$ to $\Lambda^{\leq \nu}$. By [Fiebig 2006, Proposition 3.11], the functor $\mathbb{V}$ restricts to a functor $\mathbb{V} \leq \nu: \mu_{R, \Lambda \leq \nu} \rightarrow \mathscr{V}_{R, \Lambda \leq \nu}$, which is also an equivalence of categories.

Let $\mathscr{H}_{R}^{J}$ denote the $R$-version of the category of special modules, and let $\mathscr{H}_{R, \Lambda \leq \nu}^{J}$ be the subcategory of $\mathscr{H}_{R}^{J}$ consisting of modules having support on $\mathscr{G}(\Lambda)^{\leq \nu}$. From Theorem 6.4, a module $M \in \mathscr{V}_{R, \Lambda \leq v}$ is indecomposable and projective if and only if 
there exist a $w \in \Lambda^{\leq v}$ and a $k \in \mathbb{Z}$ such that $M \cong B^{J}(w)\langle k\rangle$ and, by Proposition 6.5, there exists one and only one indecomposable $M \in \mathscr{H}_{R, \Lambda \leq \nu}^{J}$ isomorphic to $B^{J}(w)$. In summary:

Proposition 8.3. Let $P \in M_{R, \Lambda \leq v}$. Then $P$ is indecomposable, projective if and only if $\mathbb{V} P$ is an indecomposable special module.

For $\lambda_{0}$ regular, that is, $\operatorname{Stab}_{w} \lambda_{0}=\{e\}$, this was already proven in [Fiebig 2008b] and used in [Fiebig 2011], where the interchange between local and global descriptions of the image of the projective modules under $\mathbb{V}$ played a fundamental role.

\section{Acknowledgements}

I would like to acknowledge Peter Fiebig for useful discussions and Winston Fairbairn for helpful conversations. I owe many thanks to Michael Ehrig and Ben Salisbury for their careful proofreading.

\section{References}

[Beilinson and Bernstein 1981] A. Beilinson and J. Bernstein, "Localisation de $g$-modules", C. R. Acad. Sci. Paris Sér. I Math. 292:1 (1981), 15-18. MR 82k:14015 Zbl 0476.14019

[Bernstein et al. 1976] I. N. Bernstein, I. M. Gelfand, and S. I. Gelfand, "A certain category of g-modules", Funkcional. Anal. i Priložen. 10:2 (1976), 1-8. In Russian; translated in Funct. Anal. Appl. 10:2 (1976), 87-92. MR 53 \#10880 Zbl 0353.18013

[Björner and Brenti 2005] A. Björner and F. Brenti, Combinatorics of Coxeter groups, Graduate Texts in Mathematics 231, Springer, New York, 2005. MR 2006d:05001 Zbl 1110.05001

[Braden and MacPherson 2001] T. Braden and R. MacPherson, "From moment graphs to intersection cohomology”, Math. Ann. 321:3 (2001), 533-551. MR 2003g:14030 Zbl 1077.14522

[Brylinski and Kashiwara 1980] J.-L. Brylinski and M. Kashiwara, "Démonstration de la conjecture de Kazhdan-Lusztig sur les modules de Verma”, C. R. Acad. Sci. Paris Sér. A-B 291:6 (1980), A373-A376. MR 81k:17004 Zbl 0457.22012

[Deodhar 1987] V. V. Deodhar, "On some geometric aspects of Bruhat orderings, II: The parabolic analogue of Kazhdan-Lusztig polynomials", J. Algebra 111:2 (1987), 483-506. MR 89a:20054 Zbl 0656.22007

[Elias and Williamson 2014] B. Elias and G. Williamson, "The Hodge theory of Soergel biomodules", Ann. of Math. 180:3 (2014), 1089-1136. arXiv 1212.0791

[Fiebig 2003] P. Fiebig, "Centers and translation functors for the category 0 over Kac-Moody algebras", Math. Z. 243:4 (2003), 689-717. MR 2004c:17051 Zbl 1021.17007

[Fiebig 2006] P. Fiebig, "The combinatorics of category 0 over symmetrizable Kac-Moody algebras", Transform. Groups 11:1 (2006), 29-49. MR 2006k:17040 Zbl 1122.17016

[Fiebig 2008a] P. Fiebig, "Sheaves on moment graphs and a localization of Verma flags", Adv. Math. 217:2 (2008), 683-712. MR 2008m:17044 Zbl 1140.14044

[Fiebig 2008b] P. Fiebig, "The combinatorics of Coxeter categories", Trans. Amer. Math. Soc. 360:8 (2008), 4211-4233. MR 2009g:20087 Zbl 1160.20032

[Fiebig 2009] P. Fiebig, "Moment graphs in representation theory and geometry", Lecture script, 2009, http://tinyurl.com/Fiebig2009. 
[Fiebig 2011] P. Fiebig, "Sheaves on affine Schubert varieties, modular representations, and Lusztig's conjecture”, J. Amer. Math. Soc. 24:1 (2011), 133-181. MR 2012a:20072 Zbl 1270.20053

[Goresky et al. 1998] M. Goresky, R. Kottwitz, and R. MacPherson, "Equivariant cohomology, Koszul duality, and the localization theorem", Invent. Math. 131:1 (1998), 25-83. MR 99c:55009 Zbl 0897.22009

[Humphreys 1990] J. E. Humphreys, Reflection groups and Coxeter groups, Cambridge Studies in Advanced Mathematics 29, Cambridge University Press, 1990. MR 92h:20002 Zbl 0725.20028

[Kazhdan and Lusztig 1979] D. Kazhdan and G. Lusztig, "Representations of Coxeter groups and Hecke algebras”, Invent. Math. 53:2 (1979), 165-184. MR 81j:20066 Zbl 0499.20035

[Kazhdan and Lusztig 1980] D. Kazhdan and G. Lusztig, "Schubert varieties and Poincaré duality", pp. 185-203 in Geometry of the Laplace operator (Honolulu, HI, 1979), edited by R. Osserman and A. Weinstein, Proc. Sympos. Pure Math. 36, Amer. Math. Soc., Providence, R.I., 1980. MR 84g:14054 Zbl 0461.14015

[Lanini 2012] M. Lanini, "Kazhdan-Lusztig combinatorics in the moment graph setting", J. Algebra 370 (2012), 152-170. MR 2966832 Zbl 06162673

[Mazorchuk 2012] V. Mazorchuk, Lectures on algebraic categorification, Eur. Math. Soc., Zürich, 2012. MR 2918217 Zbl 1238.18001

[Mazorchuk and Stroppel 2005] V. Mazorchuk and C. Stroppel, "Translation and shuffling of projectively presentable modules and a categorification of a parabolic Hecke module", Trans. Amer. Math. Soc. 357:7 (2005), 2939-2973. MR 2006g:17012 Zbl 1095.17001

[Mazorchuk and Stroppel 2008] V. Mazorchuk and C. Stroppel, "Categorification of (induced) cell modules and the rough structure of generalised Verma modules", Adv. Math. 219:4 (2008), 1363-1426. MR 2010a:20014 Zbl 1234.17007

[Quillen 1973] D. Quillen, "Higher algebraic $K$-theory, I", pp. 85-147 in Algebraic K-theory, I: Higher $K$-theories (Seattle, WA, 1972), edited by H. Bass, Lecture Notes in Math. 341, Springer, Berlin, 1973. MR 49 \#2895 Zbl 0292.18004

[Rocha-Caridi and Wallach 1982] A. Rocha-Caridi and N. R. Wallach, "Projective modules over graded Lie algebras, I”, Math. Z. 180:2 (1982), 151-177. MR 83h:17018 Zbl 0467.17006

[Soergel 1990] W. Soergel, "Kategorie O, perverse Garben und Moduln über den Koinvarianten zur Weylgruppe", J. Amer. Math. Soc. 3:2 (1990), 421-445. MR 91e:17007 Zbl 0747.17008

[Soergel 1997] W. Soergel, "Kazhdan-Lusztig polynomials and a combinatoric[s] for tilting modules", Represent. Theory 1 (1997), 83-114. MR 98d:17026 Zbl 0886.05123

[Soergel 2007] W. Soergel, "Kazhdan-Lusztig-Polynome und unzerlegbare Bimoduln über Polynomringen”, J. Inst. Math. Jussieu 6:3 (2007), 501-525. MR 2009c:20009 Zbl 1192.20004

[Williamson 2011] G. Williamson, "Singular Soergel bimodules", Int. Math. Res. Not. 2011:20 (2011), 4555-4632. MR 2844932 Zbl 1236.18009

Received June 2, 2013. Revised July 25, 2013.

\section{MARTINA LANINI}

DEPARTMENT MATHEMATIK

FRIEDRICH-ALEXANDER-UNIVERSITÄT ERLANGEN-NÜRNBERG

CAUERSTR. 11

91058 ERLANGEN

GERMANY

lanini@math.fau.de 


\title{
PACIFIC JOURNAL OF MATHEMATICS
}

\author{
msp.org/pjm
}

Founded in 1951 by E. F. Beckenbach (1906-1982) and F. Wolf (1904-1989)

\section{EDITORS}

Don Blasius (Managing Editor)

Department of Mathematics

University of California

Los Angeles, CA 90095-1555

blasius@math.ucla.edu

\author{
Paul Balmer \\ Department of Mathematics \\ University of California \\ Los Angeles, CA 90095-1555 \\ balmer@math.ucla.edu \\ Robert Finn \\ Department of Mathematics \\ Stanford University \\ Stanford, CA 94305-2125 \\ finn@math.stanford.edu \\ Sorin Popa \\ Department of Mathematics \\ University of California \\ Los Angeles, CA 90095-1555 \\ popa@math.ucla.edu
}

\author{
Vyjayanthi Chari \\ Department of Mathematics \\ University of California \\ Riverside, CA 92521-0135 \\ chari@math.ucr.edu \\ Kefeng Liu \\ Department of Mathematics \\ University of California \\ Los Angeles, CA 90095-1555 \\ liu@math.ucla.edu \\ Jie Qing \\ Department of Mathematics \\ University of California \\ Santa Cruz, CA 95064 \\ qing@ cats.ucsc.edu
}

\section{PRODUCTION}

Silvio Levy, Scientific Editor, production@msp.org

\section{SUPPORTING INSTITUTIONS}

ACADEMIA SINICA, TAIPEI

CALIFORNIA INST. OF TECHNOLOGY

INST. DE MATEMÁTICA PURA E APLICADA

KEIO UNIVERSITY

MATH. SCIENCES RESEARCH INSTITUTE

NEW MEXICO STATE UNIV.

OREGON STATE UNIV.

\author{
STANFORD UNIVERSITY \\ UNIV. OF BRITISH COLUMBIA \\ UNIV. OF CALIFORNIA, BERKELEY \\ UNIV. OF CALIFORNIA, DAVIS \\ UNIV. OF CALIFORNIA, LOS ANGELES \\ UNIV. OF CALIFORNIA, RIVERSIDE \\ UNIV. OF CALIFORNIA, SAN DIEGO \\ UNIV. OF CALIF., SANTA BARBARA
}

\author{
Daryl Cooper \\ Department of Mathematics \\ University of California \\ Santa Barbara, CA 93106-3080 \\ cooper@math.ucsb.edu \\ Jiang-Hua Lu \\ Department of Mathematics \\ The University of Hong Kong \\ Pokfulam Rd., Hong Kong \\ jhlu@maths.hku.hk \\ Paul Yang \\ Department of Mathematics \\ Princeton University \\ Princeton NJ 08544-1000 \\ yang@math.princeton.edu
}

These supporting institutions contribute to the cost of publication of this Journal, but they are not owners or publishers and have no responsibility for its contents or policies.

See inside back cover or msp.org/pjm for submission instructions.

The subscription price for 2014 is US $\$ 410 /$ year for the electronic version, and \$535/year for print and electronic.

Subscriptions, requests for back issues and changes of subscribers address should be sent to Pacific Journal of Mathematics, P.O. Box 4163, Berkeley, CA 94704-0163, U.S.A. The Pacific Journal of Mathematics is indexed by Mathematical Reviews, Zentralblatt MATH, PASCAL CNRS Index, Referativnyi Zhurnal, Current Mathematical Publications and Web of Knowledge (Science Citation Index).

The Pacific Journal of Mathematics (ISSN 0030-8730) at the University of California, c/o Department of Mathematics, 798 Evans Hall \#3840, Berkeley, CA 94720-3840, is published twelve times a year. Periodical rate postage paid at Berkeley, CA 94704, and additional mailing offices. POSTMASTER: send address changes to Pacific Journal of Mathematics, P.O. Box 4163, Berkeley, CA 94704-0163.

PJM peer review and production are managed by EditFLOW ${ }^{\circledR}$ from Mathematical Sciences Publishers.

\section{PUBLISHED BY}

\section{mathematical sciences publishers \\ nonprofit scientific publishing}

http://msp.org/

(C) 2014 Mathematical Sciences Publishers 


\section{PACIFIC JOURNAL OF MATHEMATICS}

Volume $271 \quad$ No. $2 \quad$ October 2014

Monoids of modules and arithmetic of direct-sum decompositions

NiChOlas R. BAETH and AlFRED GEROLDINGER

On the torsion anomalous conjecture in $\mathrm{CM}$ abelian varieties

SARA CHECCOLI and EVELINA VIADA

Eigenvalue estimate and compactness for closed $f$-minimal surfaces

347

Xu Cheng, Tito Mejia and Detang Zhou

Lefschetz numbers of symplectic involutions on arithmetic groups

369

STEFFEN KIONKE

Categorification of a parabolic Hecke module via sheaves on moment 415 graphs

MARTINA LANINI

Unitary representations of $\operatorname{GL}(n, K)$ distinguished by a Galois

445 involution for a $p$-adic field $K$

NADIR MATRINGE

On $f$-biharmonic maps and $f$-biharmonic submanifolds

461

YE-LIN OU

Unitary principal series of split orthogonal groups

Alessandra Pantano, AnNegret Paul and Susana SALAMANCA RIBA 